\title{
A simple dynamical system that mimics open-flow turbulence
}

\author{
G. S. Bhat \\ R. Narasimha \\ National Aeronautical Laboratory, Bangalore 560017, India \\ S. Wiggins \\ California Institute of Technology, Pasadena, California 91125
}

Centre for Atmospheric Sciences, Indian Institute of Science, Bangalore 560012, India

(Received 7 June 1989; accepted 18 June 1990)

\begin{abstract}
The possible relevance of recent theories concerning the chaotic behavior of nonlinear dynamical systems to turbulence, especially in open flows, has frequently been questioned. Here, the issues that have led to this skepticism are investigated by studying a simple system that has been devised to include, albeit in an impressionistic way, the major mechanisms that are widely considered to operate in a broad class of turbulent flows. The variables in the system seek to represent the amplitudes of large- and small-eddy motion, respectively, and are governed by equations that allow for a Landau-Stuart nonlinear growth, a one-step Richardson cascade, and a specified time-dependent driving force. It is found that the critical value (at the onset of chaos) of the Reynolds-number-like control parameter $\left(v^{-1}\right)$ in the system depends on the character and magnitude of the driving force; and it is analytically demonstrated using the Melnikov technique that, with an appropriate choice of model parameters, chaos can persist at all sufficiently high values of the model Reynolds number (unlike as in most other low-dimensional models). The routes to chaos in the system when the forcing is increased at fixed $v$ are different from those when $v$ is decreased at fixed forcing, the latter being found to be more relevant to the case of streamwise-developing flows like a boundary layer. The observed routes are sensitive to the presence of even small stochastic components in the forcing. Computed spectral evolutions in the model show qualitative similarities with observations in boundary layer flow under different disturbance environments. It is concluded that many of the gross features of open-flow turbulence can be understood as dynamical chaos.
\end{abstract}

\section{INTRODUCTION}

\section{A. Dynamical chaos and turbulence: Some issues}

Recent developments in the theory of dynamical systems possessing strange attractors and exhibiting chaotic behavior ${ }^{1-6}$ have raised the question of whether turbulence in fluid flows could be understood as dynamical chaos. The various proposals on possible routes to chaos that have been made in these studies have found some support from observations in bounded flows such as Taylor-Couette or convection in a box. ${ }^{7-9}$ However, the existence of any connection between the dynamical chaos exhibited by such systems and fully developed turbulence, especially in open flows like boundary layers and wakes, has frequently been questioned. $^{9-11}$ It is generally felt that the chaotic phenomena observed in low-dimensional nonlinear systems may, at best, be relevant to "weak" turbulence, i.e., to stages preceding onset of fully turbulent behavior. ${ }^{12}$

The questions mentioned above crystallize into three basic issues. ${ }^{13}$

(i) Low-dimensional chaotic dynamical systems do not exhibit a strong cascade process, of the kind generally considered an essential feature of turbulence, ${ }^{14}$ where energy put in at low wave numbers or frequencies produces strong fluctuations at very high wave numbers or frequencies. Thus, while the spectral density in the well-known model of Lorenz ${ }^{15}$ is exponential in the frequency and drops by about six decades over a frequency range of one to ten, the corresponding drop in a typical shear flow ${ }^{16}$ is only about a decade (see Fig. 1) for the same frequency range. In other words, the dynamical chaos frequently observed tends to be slow, as may be expected from the wide prevalence of period-doubling bifurcations.

(ii) In the absence of a special relaminarizing agency, ${ }^{17}$ turbulence invariably persists in the flow for all values of the control parameter beyond that at onset, whereas in low-dimensional dynamical systems chaos and order often alternate in narrow windows, and indeed chaos eventually tends to disappear as the control parameter is increased (the Lorenz system is again a good example; see, e.g., Sparrow ${ }^{18}$ ). It is not clear whether higher-order truncation will solve the problem. In two-dimensional Bénard convection, Curry et al. ${ }^{19}$ found that chaotic behavior disappears when a large number of modes are considered, and concluded that the chaos observed in earlier models was due to severe truncation, i.e., lack of sufficient resolution. On the other hand, solutions of the Navier-Stokes equations in a two-torus suggest the opposite; in a five-mode truncation ${ }^{20}$ chaos disappeared for large values of the Reynolds number, whereas in models truncated at seven or more modes ${ }^{21}$ chaos seemed to persist once it had set in.

(iii) In the dynamical systems studied to date, the onset of chaos occurs (for each system) at some fixed value 


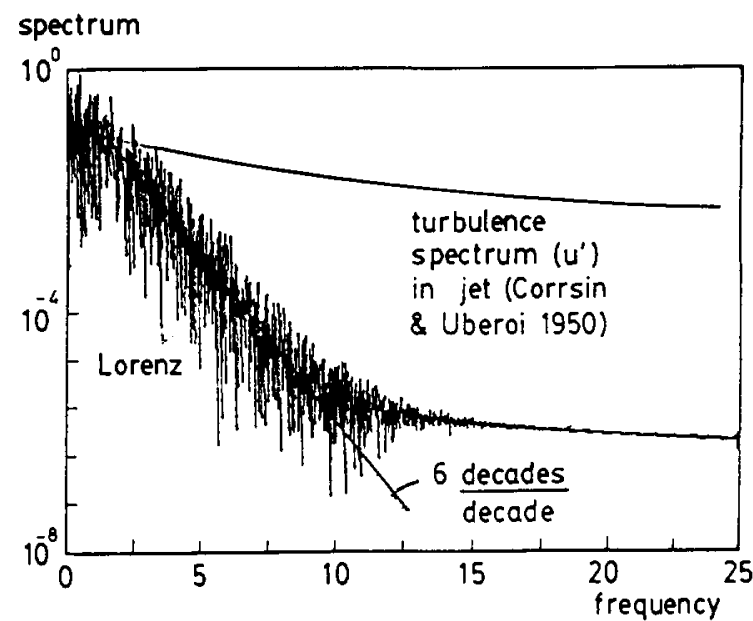

FIG. 1. Comparison of a typical spectrum of the solution of the Lorenz mode ${ }^{15}$ with that of the streamwise fluctuations in a turbulent jet, ${ }^{16}$ normalized to agree at the lowest frequencies. The flat spectrum of the Lorenz solution beyond a frequency of 10 is a numerical artifact.

of the control parameter, whereas it is widely recognized that in many open or semiopen flows (particularly in boundary layers and ducts) the onset of transition is governed by external or environmental disturbances; indeed there is solid experimental evidence (e.g., Schubauer and Skramstad ${ }^{22}$ ) to show that transition to turbulence in boundary layers can be delayed enormously by reducing the level of such disturbances, leading to the view that there is no such thing as "free" transition in these flows. Indeed, recent analysis of available experimental data on boundary layers that allows for the presence of residual nonturbulent disturbances in the facilities used for testing strongly suggests ${ }^{23}$ that the Reynolds number at transition to turbulence is inversely proportional to the disturbance intensity (Fig. 2), at least over the (fairly wide) range of disturbance levels covered in experiments to date.

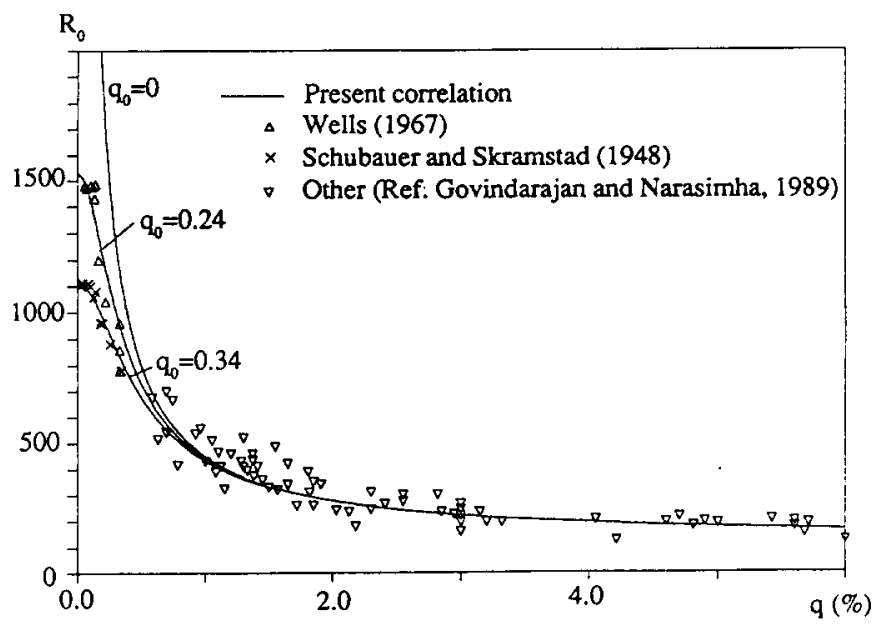

FIG. 2. Dependence of transition Reynolds number $R_{t}$ in a boundary layer on free-stream turbulence $q$; at low values of $q$ transition is driven by facility-specific nonturbulent disturbances, parametrized by the variable $q_{0}$. The full lines show a correlation with $\left(q^{2}+q_{0}^{2}\right)^{1 / 2}$ proposed in Ref. 23 .
In an early review of the new ideas, Monin ${ }^{10}$ forcefully made the criticism listed as (ii) above, but in addition suggested that the presence of only a small number of spatial modes in the nonlinear systems considered (e.g., that of Lorenz) was a serious deficiency, for this implied that there was no continuous spectrum in wave number, i.e., no spatial chaos, of the kind considered essential for flow turbulence. However, the latter criticism will now appear less fundamental than the others, in part because of the discovery of strong spatial order in fully turbulent flows (e.g., Brown and Roshko ${ }^{24}$ ) and in part because the spatial chaos can be generated through mere advection (e.g., Aref $^{25}$ ). Furthermore, temporal development in a Lagrangian frame can approximate spatial development in an Eulerian frame, especially in convectively unstable flows.

The question that arises, therefore, is how generally the criticisms (i)-(iii) apply to dynamical systems. Because of the difficulty of analyzing the complete Navier-Stokes equations, several attempts have been made to simulate aspects of flow turbulence in simpler models, beginning with such celebrated efforts as those of Burgers ${ }^{26}$ and Lorenz. ${ }^{15}$ Other models that have attracted considerable attention are the Ginzburg-Landau ${ }^{27}$ and Sivashinsky ${ }^{28}$ equations. A significant advance has been made recently by Aubry et al. ${ }^{29}$ in linking relatively low-dimensional chaotic dynamics and turbulence in open-flow systems. They model the behavior of streamwise vortex rolls near the wall in a fully turbulent boundary layer and show that it is possible to capture the ejections and bursting events observed experimentally. ${ }^{30}$ Their study, utilizing modes derived from observation but incorporating Navier-Stokes dynamics, confines itself by design to a specific flow, and in particular is not concerned with transition; thus the Reynolds number does not explicitly appear as a parameter in the model.

However, none of these interesting efforts directly addresses the criticisms listed above.

Some very interesting experimental work has been reported on wakes; ${ }^{31-33}$ the central issue here appears to be the necessity or otherwise for the presence of forcing of some kind to induce chaos.

In the present paper we briefly describe one of a set of simple models devised by Narasimha and Bhat, ${ }^{34}$ and demonstrate that its behavior answers the above criticisms and mimics certain generic features of open-flow turbulence. The model is "impressionistic" (in the sense used in Ref. 35 ), being intended to offer insight rather than quantitative predictions for any particular flow, although it is motivated by the considerable experimental data available on boundary layer transition. The main advantage in the study of such impressionistic models is the elucidation of basic issues, as the complexities not relevant to the resolution of such issues can be eliminated. It is hoped that the conclusions so drawn will be of use in the formulation or study of more exact models for turbulent flow.

In view of the emphasis in the rest of the paper on the routes to chaos in the model, a brief review of the relevant work seems appropriate. 


\section{B. Routes to chaos: A brief survey}

Extensive studies have been made of various "scenarios" that lead to chaotic behavior in dynamical systems." In general, a system that is at rest at low values of the control parameter $\mu$ loses stability when $\mu$ increases, and exhibits a steady behavior; for higher $\mu$, the steady solution loses stability leading to a periodic (limit-cycle) behavior through a Hopf bifurcation (change from one steady solution to another is also possible before periodic behavior ${ }^{1}$ ). As $\mu$ increases further the limit cycle may lose stability, after which different routes to chaos are possible.

Some of these routes are best understood from maps, but they are equally valid for differential equations that can be investigated through the maps that result from taking Poincaré sections. For any such map its fixed points and their stability determine how the limit cycle undergoes transition to chaos. For example, if the fixed point (which in the map represents limit-cycle behavior) has an eigenvalue of -1 at some value of $\mu=\mu^{\prime}$, then for $\mu>\mu^{\prime}$ the system undergoes a cascade of period-doubling bifurcations, which, in one-dimensional hump maps that are quadratic near the hump, exhibit certain universal characteristics described through the Feigenbaum numbers. ${ }^{3}$ The cascade ends in an accumulation point, usually denoted by $\mu_{\infty}$, at which the system undergoes an infinite number of bifurcations. At $\mu=\mu_{\infty}$, the system is expected to exhibit a periodic behavior but does not possess a wide band spectrum, which is encountered only for $\mu>\mu_{\infty}$. This scenario is well tested in both numerical and physical experiments.

If, on the other hand, the fixed point has two distinct (complex) eigenvalues with an absolute value of 1 at some value of $\mu=\mu^{\prime}$, then for $\mu>\mu^{\prime}$ stability is lost through a Hopf bifurcation giving a limit cycle in the map, or motion on a two-torus $T^{2}$ that is quasiperiodic with two incommensurate frequencies in the original system. If $\mu$ increases further, the torus $T^{2}$ may lose stability giving a three-torus $T^{3}$ and so on. Generalizing this scenario, Landau ${ }^{36}$ proposed that turbulence is a quasiperiodic motion with a large number of incommensurate frequencies.

It has, however, been shown ${ }^{1,2}$ that a torus $T^{k}$ with $k \geqslant 3$ is structurally unstable and that a "strange attractor" could result, with sensitive dependence of the solution on initial conditions. In this scenario (called RTN, after Ruell, Takens, and Newhouse) the power spectrum exhibits first one, then two, and possibly three independent frequencies before a wide band spectrum appears. Some experiments ${ }^{37}$ have shown that when the third frequency is about to appear, the spectrum develops a broadband consistent with the above scenario.

A modification of this scenario often seen in many systems (typically in periodically forced ones) involves quasiperiodic motion and frequency locking followed by sudden chaos. ${ }^{38}$ A simple example is provided by the circle $\operatorname{map}^{39}$

$$
\theta_{n+1}=\theta_{n}+(F / 2 \pi) \sin 2 \pi \theta_{n}+\Omega, \quad 0 \leqslant \theta<1,
$$

which maps the circumference of a circle to itself. Here the sinusoidal term represents the effect of periodic forcing, $\Omega$ represents the ratio of the natural frequency to the forcing frequency, and $F$ is the strength of the nonlinearity. For small values of $F$ quasiperiodic solutions are likely, and as $F$ approaches 1 frequency locking is more likely. For $F>1$, the map becomes noninvertible. The boundary $F=1$ represents transient chaos; for larger $F$ steady chaotic solutions are possible. ${ }^{38,40}$

Another widely studied scenario is that of intermittency, first described by Pomeau and Manneville. ${ }^{41}$ This occurs if the map attains a slope of +1 at some value of $\mu$ ( $=\mu^{\prime}$, say); beyond this value solutions show small amplitude oscillations interrupted by bursts that become more frequent as $\mu$ increases. Physically this corresponds to a stable fixed point meeting an unstable fixed point leading to their total disappearance for $\mu>\mu^{\prime}$ (saddle-node bifurcation). Such intermittency has been observed in the Lorenz model, ${ }^{41}$ interestingly at a value of $r$ (the normalized Rayleigh number in the model) near 166 , much larger than the value of 24.06, at which chaos is first encountered in the model.

It may be noted here that all chaotic phenomena known today are related to homoclinic or heteroclinic connections. $^{6}$ Yorke and Aligood ${ }^{42}$ have shown that perioddoubling bifurcations lead to homoclinic connections (i.e., stable and unstable manifolds of the same fixed point intersect each other) and horseshoes in the attractor. Ostlund et al. ${ }^{40}$ have shown that chaos from quasiperiodicity and a frequency-locked state results from intersection of stable and unstable manifolds of separate fixed points in a Poincaré map, i.e., from heteroclinic connection.

Although chaos can arise in purely deterministic systems, most real open-flow systems are subjected to noise in some form or other, so it is important to understand the influence of (partially and wholly) stochastic forcing. In a study of the forced dissipative motion of an anharmonic oscillator, ${ }^{43}$ it has been shown that, with sinusoidal forcing, the system undergoes period-doubling bifurcations before the onset of chaos as the forcing frequency is lowered; in the presence of noise, the threshold frequency (i.e., the frequency at the onset of chaos) increases almost linearly with the noise level and in effect produces a bifurcation gap in the set of available states. In the logistic map, for example, higher periodicities disappear in the presence of noise as a result of the spreading of iterates of the map and consequent merging of close-by islands (regions) of attraction. $^{44}$ Universal scaling laws near transition have been found for the Lyapunov characteristic exponent in a system that follows a period-doubling route, and for the average duration of small amplitude ("laminar") motion in systems exhibiting intermittency.

It is not very clear what happens to the quasiperiodicity route to chaos in the presence of noise, but the expectation is that the influence is not strong. ${ }^{4}$

While the studies cited above support the general view that noise destabilizes a system, Matsumoto and Tsuda ${ }^{45}$ show that noise can, under certain conditions, induce some order in certain types of maps, e.g., those with flat tops and steep gradients.

A large number of well-controlled experiments in fluid 
flow have been carried out to test the predictions of $d y$ namical theory. Gollub and Swinney ${ }^{37}$ find that in TaylorCouette flow, the observed transition sequence as Reynolds number increases is: no motion $\rightarrow$ steady motion $\rightarrow$ periodic state $\rightarrow$ two frequencies $\rightarrow$ disappearance of frequency and appearance of new frequency $\rightarrow$ sudden disappearance of sharp peaks, emergence of a broadband spectrum. This route supports the RTN scenario. Gollub and Benson ${ }^{7}$ carried out detailed experiments on convection in a small cell with different aspect ratios and Prandtl numbers Pr, and observed four different types of transition: (i) Quasiperiodic motion at two frequencies leading to phase locking or entrainment, and chaos as a result of loss of entrainment. (ii) Cascade of period-doubling bifurcations. (iii) Three incommensurate frequencies before the appearance of a broadband spectrum. (iv) Intermittency. A feature of these experiments was that the behavior was sensitive to small changes in $\operatorname{Pr}$ and aspect ratio.

In a convection experiment carried out with silicon oil as working fluid $(\operatorname{Pr}=130)$, Berge et al ${ }^{46}$ observed bursts of strong perturbations at random intervals between normal oscillations (laminar periods), consistent with the scenario proposed by Pomeau and Manneville. ${ }^{41}$

Giglio et al., ${ }^{47}$ studying convection in a small cell, could detect subharmonics up to the fourth order; beyond this the system seemed to deviate from the Feigenbaum scenario. Libchaber et al. ${ }^{48}$ using mercury as a working fluid, applied a magnetic field along the axis of the convective rolls to give convection a better two-dimensional character. They observed period-doubling bifurcations and subharmonics up to the fifth order following the Feigenbaum scenario. In another experiment, ${ }^{49}$ a low Prandtl number fluid developed two-dimensional cells with the scenario two frequencies $\rightarrow$ frequency locking $\rightarrow$ period-doubling bifurcations, whereas high Prandtl number fluids had a three-dimensional character and followed either the route two frequencies $\rightarrow$ three frequencies $\rightarrow$ chaos or the route two frequencies $\rightarrow$ intermittency $\rightarrow$ chaos.

We now briefly discuss the model proposed by Narasimha and Bhat ${ }^{34}$ before studying the routes to chaos in the model.

\section{THE MODEL}

The basic philosophy in constructing the model studied here was to incorporate in it those physical mechanisms that appear essential to turbulent behavior, at the same time retaining simplicity to enable detailed analysis. The conventional method of using a truncated Galerkin approximation to obtain a set of ordinary differential equations from the governing partial differential equations will, in general, need a large number of modes, and hence also equations, to even attempt meeting the criticisms of Sec. I. The present model avoids this difficulty by treating turbulent flow as mainly the outcome of interaction between motions at two widely different scales, somewhat in the spirit of Liepmann's "turbular" fluid, ${ }^{50}$ the emphasis being not on any particular flow, such as over a flat plate or behind a cylinder, but on general physical arguments valid for a wide class of flows. More specifically, the spectral or

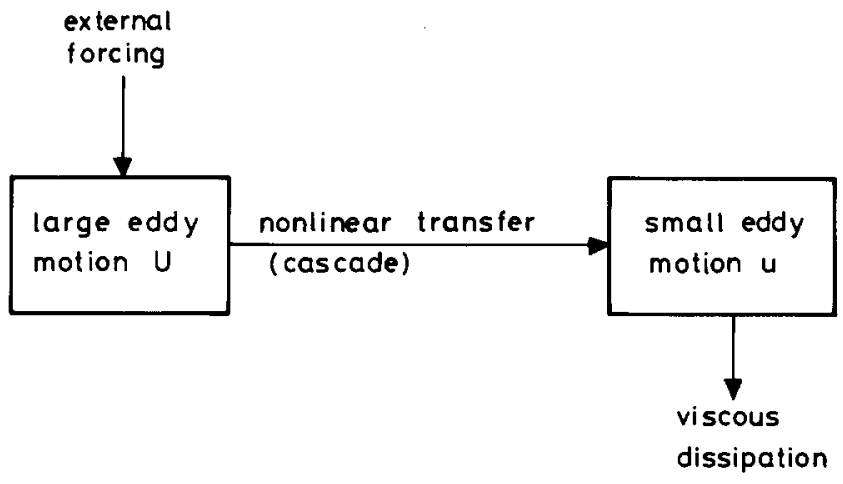

FIG. 3. Schematic representation of energy transfer in a turbulent flow by a single-step cascade.

wave number space is considered to consist of two broad regions, one where nonlinearity and external disturbances play the major role (representing the so-called large-eddy motion) and the other where direct viscous dissipation is significant, representing the small or Kolmogorov-scale motion; these two scales are coupled by a nonlinear energy transfer mechanism, often called the Richardson cascade process, schematically illustrated in Fig. 3.

The model has two independent variables $U, u$ both considered functions only of time $t$, and a specified external forcing $q(t)$. Note that $U$ and $u$ may be thought of as representing the amplitudes characteristic of large and small eddies, respectively, the actual velocity being a combination like

$$
a U(t) \exp i\left(\mathbf{k}_{1} \cdot \mathbf{x}-\omega_{1} t\right)+b u(t) \exp i\left(\mathbf{k}_{2} \cdot \mathbf{x}_{2}-\omega_{2} t+\phi\right),
$$

where $k_{1}, \omega_{1}$ and $k_{2}, \omega_{2}$ are characteristic large- and smalleddy wave numbers and frequencies and $a$ and $b$ are weighting functions that are a measure of the bandwidth covered by the respective motions in wave number space. For the sake of simplicity we shall assume that the eddies have a common celerity, $\omega_{1} / k_{1}=\omega_{2} / k_{2}$, and that time is measured in a coordinate frame moving with the fluid at this speed, invoking a form of Taylor's hypothesis. Note the significant departure from earlier studies, typified by the Lorenz system, where only the lowest few modes are selected for describing the dynamics. If $u(t)$ is seen as including Kolmogorov-scale motions, we should expect the weighting factor $b$ to be a function of the Reynolds number characterizing the large-eddy motion. ${ }^{14}$ An alternative interpretation that is helpful in motivating the model is inspired by experimental observations in boundary layer transition, ${ }^{51,52}$ where it is found that as the amplitude of oscillation in the primary Tollmien-Schlichting instability increases, an intense small-scale high-shear layer develops; the instability of this layer rapidly leads to the growth of fine-scale motion. In Gaster's experiments ${ }^{53,54}$ on spectral evolution in such a transitional boundary layer, a clear distinction can be made between the development of slow (or low frequency) and fast (or high frequency) chaos. There is here, once again, a natural division into two widely different scales. 
The importance of including a forcing term $q(t)$ is evident from experimental data on boundary layers. ${ }^{11,53}$ It is our premise here that in boundary layers certainly, and possibly in most open flows, there is no transition if there is no forcing (ignoring microscopic triggering such as that resulting from Brownian fluctuations): the flow is seen as a (somewhat peculiar) driven nonlinear oscillator. (As an aside, therefore, one way of keeping a flow laminar would be to provide it with a quiet environmental sheath, itself maintained possibly by active control operating on the receptive frequency bands.)

The following physical considerations, especially those relevant to boundary-layer flow, were used to suggest possible forms for the model.

(i) The large-scale variable $U$ must be governed by a control parameter that plays the role of Reynolds number (denoted by $R$, say), whose variation changes overall system behavior.

(ii) There must be a critical value $R_{c}$ of this parameter such that for $R<R_{c}$ the motion is stable, and for $R$ $>R_{c}$ the system exhibits linear instability.

(iii) For $R>R_{c}$ the growth of $U$ because of linear instability will eventually be checked by nonlinearity and saturate at some finite maximum that depends on $R$.

(iv) The value of $R$ at which onset of chaos will occur, say $R_{t}$, will in general be higher than $R_{c}$ and depend on the forcing $q(t)$ (which may be deterministic or stochastic or a combination of both).

(v) The small-scale motion $u$ will gain energy from the large-scale motion $U$ by nonlinear interaction.

(vi) Energy at the small scales is lost by direct viscous action.

Clearly, not all these considerations are valid for all open flows (e.g., pipe flow does not exhibit linear instability at all), and some of them (e.g., linear instability followed by nonlinear saturation) are present in earlier models. ${ }^{27-29}$ (A distinction may have to be made between convectively and absolutely unstable flows, ${ }^{55}$ but as the distinction is not Galilean invariant the observer's coordinate frame would have to be specified carefully.) Nevertheless, a model that successfully reproduces the behavior described previously will cover a fairly wide class of open flows, and can be easily modified to mimic others if necessary.

Several nonlinear models may be written down that are generally consistent with the features listed above. Here we shall discuss the model called system 2 in Ref. 34, as it was found to be the simplest model that has many desirable and interesting properties. The model is described by the equations

$$
\begin{aligned}
& \frac{d U}{d t}=U\left(1-v-U^{2}\right)-K u|u|+q(t), \\
& \frac{d u}{d t}=k U(|u|+\sigma)-\hat{v} u,
\end{aligned}
$$

with the forcing taken in general to be the sum

$$
q(t)=\bar{q} \cos \omega t+q_{n} \xi(t),
$$

where $\bar{q}$ is the amplitude of a periodic component at frequency $\omega$ and $\xi(t)$ is a stochastic term with zero mean and unit mean square.

It is necessary to discuss briefly the different terms in Eqs. (3). First of all, the linear and cubic terms in $U$ in (3a) are prompted by the argument of Landau ${ }^{36}$ and the nonlinear stability theory of Stuart. ${ }^{56}$ The parameter $v$ represents the effect of viscosity on the large-scale motion. With an expression like (2) for the velocity, the viscous term in the Navier-Stokes equation will be proportional to the actual viscosity of the fluid times $k_{1}^{2}$ and $k_{2}^{2}$; the $v$ and $\hat{v}$ of (3) are to be thought of as these products. The limil $v \rightarrow 0$ is like the Reynolds number tending to infinity in a flow, and (3a) reflects the fact that at high Reynolds numbers nonlinear interaction dominates over viscous effects on the large scales (cf. the principle of high Reynolds number similarity, ${ }^{57}$ according to which the viscosity is asymptotically irrelevant in large-eddy motion as the flow Reynolds number tends to infinity). Clearly $v$ plays the role of control parameter here and may be thought of as proportional to the inverse of a Reynolds number based on largeeddy scales. The coefficients $K$ and $k$ govern the nonlinear interaction between large and small-eddy motion, and represent as a single-step process the net effect of the cascade, which actually takes place through intermediate scales. (The picture implicit in the model is that these intermediate scales are "dummies" that constitute a conveyer belt carrying energy from the large to the small eddies.) If Eqs. (3a) and (3b) are multiplied, respectively, by $U$, $u$, we obtain equations for the rate of change of the energies $U^{2}$, $u^{2}$, which show that the loss from the large eddies, $K U u|u|$, can exactly balance the gain by the small eddies $k U u|u|$ if $a^{2} K=b^{2} k$. The small-eddy variable $u$ grows because of the interaction with $U$ and is assumed to act roughly like a Reynolds stress on $U$, as given by the second term in (3a). The parameter $\sigma$, assumed small, is added to ensure that $u$ is always excited in the presence of $U$. We have found that it plays no great role in the model, but in its absence $u=0$ is always a solution. In practice, numerical noise ensures that $u$ does not remain zero even if $\sigma=0$, but we have preferred to put in explicitly an "agitation" from the eddies instead of leaving it to numerical "stirring." At the small scales, viscous dissipation is represented by the term containing $\hat{v}$, which is assumed to be significant always and in particular, therefore, does not vanish with $v\left(k_{2}\right.$ adjusting itself suitably in the limit); this is a crucial feature of the present approach.

The forcing (3c) permits us to mimic experimental studies undertaken to elucidate the mechanisms underlying transition, where free-stream turbulence and other stochastic disturbances have often been greatly reduced and artificial periodic forcing introduced. ${ }^{22}$

The use of the absolute value sign in (3) introduces a symmetry in the $(U, u)$ phase plane [the transformation $U \rightarrow-U, u \rightarrow-u$ leaves Eqs. (3a) and (3b) invariant], and actually results in a simpler system (with only three fixed points instead of five).

We look upon all variables in Eqs. (3) as being nondimensional, the scales adopted for the purpose being the 
saturating large-eddy velocity amplitude and the $e$-folding time in the linear instability constituting the LandauStuart part of Eq. (3a) in the limit $v \rightarrow 0$. The parameter $v$ itself is best seen as the ratio $R_{c} / R$.

We believe that the system ( 3 ) is the simplest impressionistic model that can be devised for a wide class of driven fluid-dynamical oscillators. It shares some characteristics with other widely studied nonlinear oscillators (e.g., the Duffing ${ }^{58}$ ), but differs from them in several respects, in particular, by including a model for the cascade process that is such a distinguishing characteristic of fluid flows.

It is seen that, apart from the forcing, there are five unspecified parameters in the model. Out of these $\sigma$ is assumed small and has been assigned a value of 0.05 in all the investigations reported here. The choice of values for the other parameters is guided by the solutions of the unforced system, which we now briefly describe (details are available in Refs. 59 and 60 ).

\section{UNFORCED SOLUTIONS}

In the absence of forcing, the system has only two dependent variables; trajectories in the $U-u$ phase plane cannot cross each other, and chaotic behavior is not possible.

\section{A. The fixed points}

The fixed points $\left(U_{*}, u_{*}\right)$ are the solutions of $d U / d t=0=d u / d t$. The origin is clearly one fixed point (say $P_{0}$ ). The others are given by the equations

$$
\begin{aligned}
u_{*}= & \sigma k U_{*} /\left(\hat{v}-k U_{*} S\right), \\
U_{*}^{4} & -2 \delta U_{*}^{3} S-\left(1-v-\delta^{2}\right) U_{*}^{2}+\left[2 \delta(1-v)+K \sigma^{2}\right] \\
& \times U_{*} S-(1-v) \delta^{2}=0,
\end{aligned}
$$

where $S=\operatorname{sgn} u_{*}$ and $\delta=\widehat{v} / k$. As $K$ is multiplied by $\sigma^{2}$ and $\sigma$ is assumed to be small, the dependence of $U_{*}$ on $K$ (unless it assumes very large values) is not as significant as that on $v$ and $\delta$. Now $\delta$ clearly represents the ratio of viscous dissipation to nonlinear energy transfer; as there is a balance between the two in a turbulent flow (the viscous scales adjusting themselves to dissipate the energy that cascades down from the large eddies), we shall require $\delta$ to be of order unity.

With $\delta=O(1),(5)$ has no real roots for $v>1$; for $v<1$, there are two real roots with the same magnitude but of opposite signs. Therefore at $v=1$ there is a pitchfork bifurcation, giving rise to two additional fixed points for $v<1$, to be labeled $P_{+}$and $P_{-}$, situated, respectively, in the first and third quadrants of the phase plane. Their variation with $v$ for the "standard" system parameters we shall adopt (for reasons to be set forth below), namely $\delta=0.55633$ and $K=k=2.3$ ( $\sigma$ has been fixed at the value 0.05 , as was already mentioned), is shown in Fig. 4. It is seen that as $v \rightarrow 0$ the position of the large-eddy fixed point $U_{*}$ is insensitive to $v$, which is consistent with the principle of high Reynolds number similarity. ${ }^{57}$

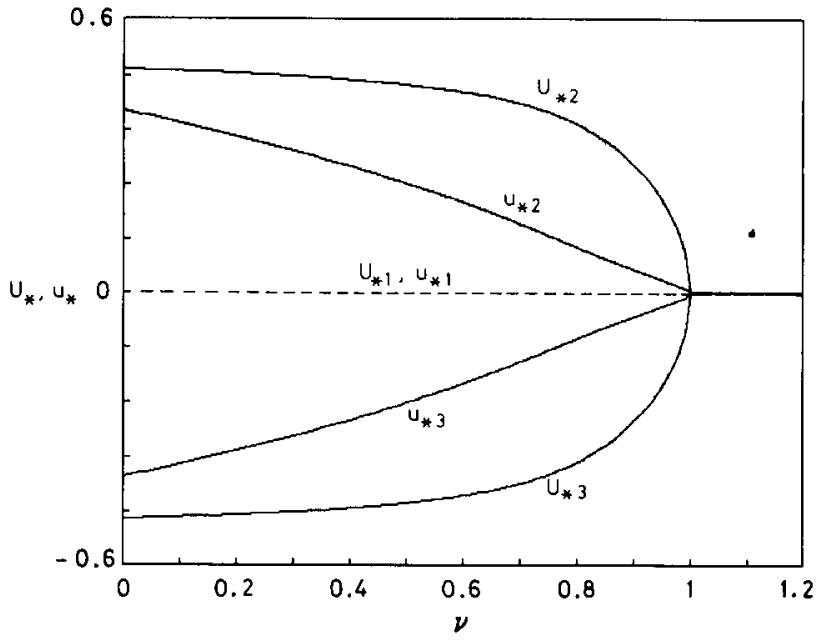

FIG. 4. Dependence of the fixed points $U_{*}$ and $u_{*}$ on $v$ for standard values of the system parameters.

\section{B. Stability of the fixed points}

The origin, with eigenvalues $1-v$ and $-\hat{v}$, is a stable node for $v>1$; after the pitchfork bifurcation at $v=1$, it becomes a saddle point for $v<1$. This is the basis for the suggestion that the value $v=1$ corresponds to the instability-critical Reynolds number $R_{c}$ of a flow (not to be confused with the transition Reynolds number), so that we may put $v=R_{c} / R$. The other fixed points $P_{ \pm}$are stable nodes or foci for $v$ near 1 , but they lose stability through a Hopf bifurcation at some value of $v,=v_{H}$ say $(<1)$, which depends on $v$ and $\hat{v}$ for $K=k=2.3$ in the manner shown in Fig. 5. For $v<v_{H}, P_{ \pm}$are unstable. ${ }^{59}$

The fixed points and their stability are not significantly influenced by values of $K$ not too different from that of $k$. In view of this, here we have taken $k=K$ to reduce the

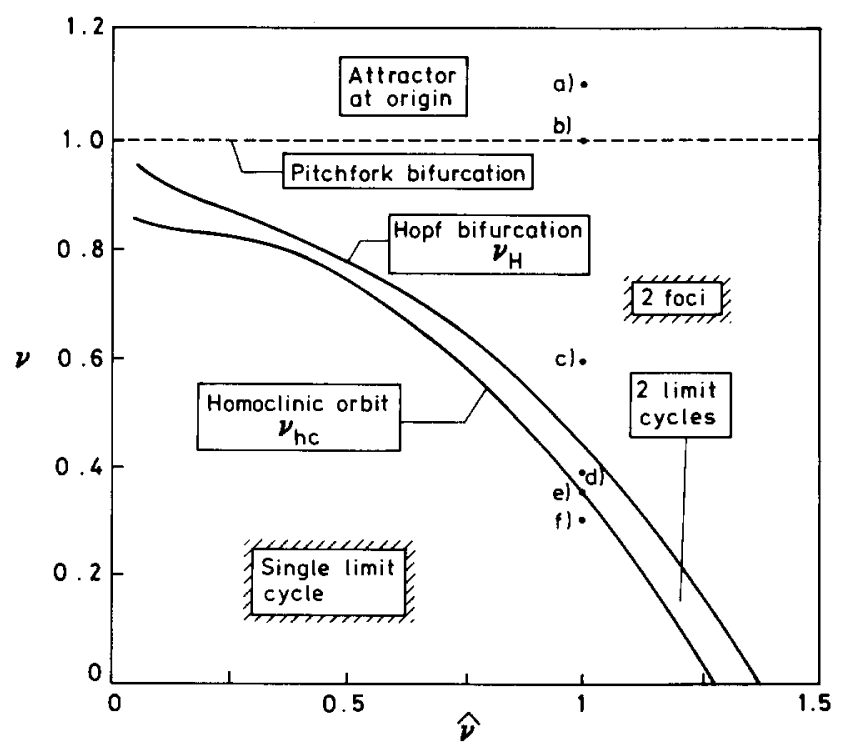

FIG. 5. Bifurcation boundaries in the unforced system as a function of $v$ and $\hat{v}$ for $K=k=2.3$. 
(a)

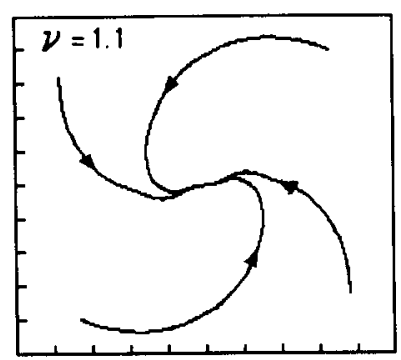

(c)

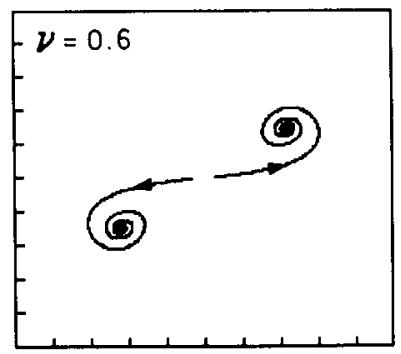

(e)

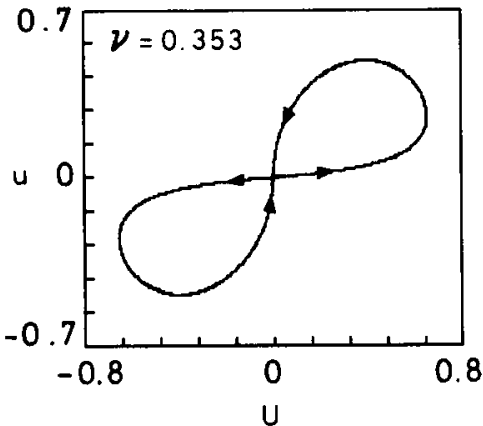

FIG. 6. Typical phase portraits at different values of $v$ labeled with the same letters as in the section in Fig. 5; $\widehat{v}=1$.

number of independent parameters in the system. The results for forced solutions are not sensitive to this assumption provided $K$ does not differ substantially from $k$.

\section{Attractors in the unforced system}

The nature of the attractors in the unforced system is illustrated in Fig. 6 for the nonstandard case $\hat{v}=1$. For $v>1$, the origin is the sole attractor; for $1>v>v_{H}$ ( $\approx 0.44$ ) the other two fixed points become attractors, and these lose stability to give limit cycles for $v<v_{H}$. As $v$ decreases further, each limit cycle grows in phase space, and a special case occurs if the limit cycle touches the origin, resulting in a saddle fixed point being connected to itself, i.e., in a homoclinic orbit. The value of $v$ (denoted by $v_{\text {hc }}$ ) at which this happens in the system depends on $\hat{v}$, as shown in Fig. 5.

Now a homoclinic orbit plays a special role in dynamical systems because it can break even under very small perturbations, giving rise to chaos. Since it should take little external forcing to trigger turbulence at high Reynolds numbers, we should want the present model to become very sensitive to external forcing as $v \rightarrow 0$. We there- fore assign that value for $\hat{v}$ that ensures a homoclinic orbit at $v=0$ for the selected $K$.

In the computations reported below, we have selected $K=k=2.3$ and $\hat{v}=1.27956$ (corresponding to $\delta=0.55633$ ) as "standard values," satisfying the above criterion. It is worth reiterating that the values of $K, k$, and $\sigma$ can be chosen over a fairly wide range without altering the qualitative behavior of the system significantly, but for any set that is chosen the value of $\hat{v}$ assigned is of crucial importance.

With this choice, $v$ and $q(t)$ are the only parameters now left free in the model.

The numerical results presented below were obtained by integrating the equations in double precision on a VAX11 using a code discussed in Ref. 59. This code adjusts the order of an interpolating polynomial and the step size in time to control the local error in such a way that increasing the order does not change the values at the desired output time by more than the specified accuracy level. This accuracy level has ranged, depending on the delicacy of the conclusions drawn, from $10^{-5}$ to $10^{-8}$ between outputs sampled at time intervals from 0.05 to 1.0 .

\section{Physical interpretation of unforced solutions}

As we shall demonstrate later, the nature of the solutions of the forced system, even when chaotic, is greatly influenced by the motion around the fixed points of the unforced system. These may be thought of as representing states of flow that the system prefers in some way, although the strength of the preference depends on system parameters. The presence of two fixed points, apart from the origin, indicates the existence of two such preferred states of motion at $v<1$. There are many flows that exhibit such behavior. For example, the wake behind a body (especially one that is blunt) contains vortices of opposite sign in a Karman vortex street when the Reynolds number is not too low; organized vortices of some kind are known to persist in the wake even at very high Reynolds numbers. ${ }^{61,62}$ It has been shown by Sreenivasan et al. ${ }^{63}$ that the Karman vortices are the result of a Hopf bifurcation at an initial Reynolds number of order 40 , followed first by exponential growth in a linear regime and later by nonlinear saturation as in the Landau-Stuart equation-both of these mechanisms being present in Eq. (3a). These must, however, be distinguished from a convective far-wake instability, which appears to need external forcing for its maintenance. ${ }^{55,64}$ As a second example, we may cite the (turbulent) boundary layer in which the flow may largely be described as the succession of two basic patterns of motion called, respectively, "sweeps" and "ejections,",30 the latter occurring either singly or in trains from multiple streaks. $^{65}$

It is therefore useful to think of the two nonzero fixed points in the present dynamical system as indicating the possibility of two characteristic patterns of motion, i.e., the fixed points may be regarded as representing latent coherent structures. 


\section{FORCED SOLUTIONS}

With time-dependent forcing, the direction of the trajectory at any phase point can vary with $t$, giving rise to the possibility that trajectories can cross each other in the phase plane. We first study a purely sinusoidal forcing function $\left[q_{n}=0\right.$ in $\left.(3 \mathrm{c})\right]$, and shall show, numerically as well as analytically, that the resulting deterministic system does indeed possess chaotic solutions under certain conditions.

\section{A. Evidence of chaotic behavior}

Here by chaos we shall mean the disappearance of the autocorrelation at sufficiently large time lags, or the emergence of a broadband spectrum. Such chaos is known to appear from the irregular behavior of deterministic systems as a result of repeated stretching and folding of volumes in phase space, ${ }^{6}$ with the associated property of sensitive dependence on initial conditions. We shall analytically demonstrate below that the present system exhibits chaotic behavior in a certain limit by the use of a perturbation technique of Melnikov. ${ }^{6,66}$ More generally, however, inferences about the existence of chaos have to be made from numerical studies.

We begin by showing a typical set of Poincaré maps for fixed $v(=0.1)$ and forcing frequency $\omega(=1)$ as the forcing amplitude is increased (Fig. 7). These maps are obtained by sampling the solution of (3) at time intervals equal to the period of forcing, i.e., successive points are iterates or images of their predecessors one period earlier. (In spirit this procedure bears some resemblance to the technique of conditional sampling used in the search for persistent "coherent" structures in turbulent flows.) The single fixed point in Fig. 7 at $\bar{q}=0.070$ represents periodic behavior at the forcing period. At $\bar{q}=0.078$ there are two points in the plot, indicating that the orbit returns to the same point after every two forcing periods, i.e., that the period of the oscillation has doubled. As $\bar{q}$ increases further more period-doubling bifurcations take place, and by $\bar{q}=0.081$ the Poincare section shows a folded and layered structure, which is a well-known signature of chaotic behavior. $^{5}$ In this particular case the onset of chaos is clearly preceded by a cascade of period-doubling bifurcations, but we will return to a more detailed discussion of the routes to chaos in Sec. V.

Now it is well known ${ }^{5,67}$ that chaotic behavior is in general the result of an intersection of the stable and unstable manifolds associated with hyperbolic invariant sets such as a fixed point, periodic orbit or invariant torus, resulting in a structure that has homoclinic tangles and exhibits properties associated with Smale horseshoes. Iterations of a map possessing Smale horseshoes can be shown to be equivalent to the Bernoulli shift process that can be proved to possess chaotic behavior. ${ }^{6}$ Therefore establishing that manifolds intersect is a conclusive step in demonstrating that the system is chaotic. We now do this for the present model by the Melnikov technique.
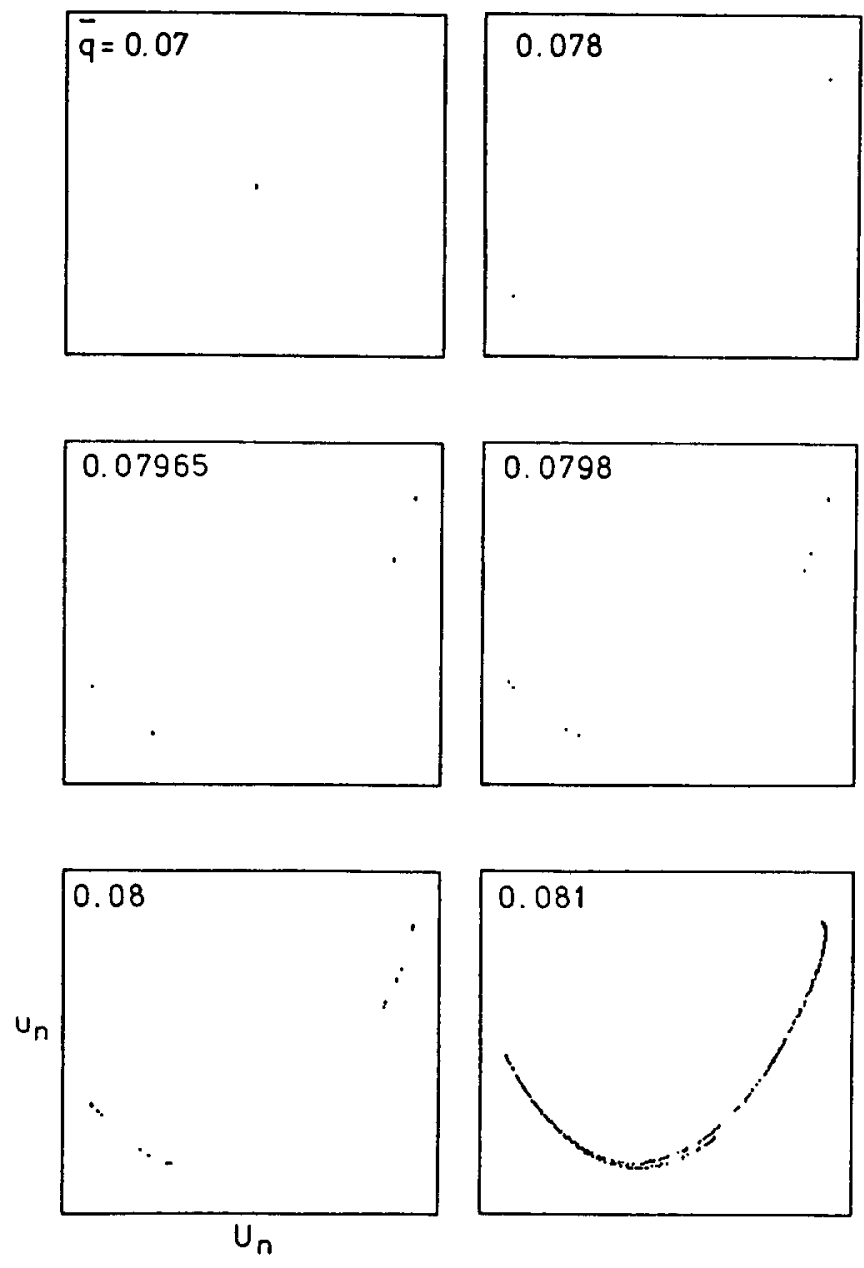

FIG. 7. Poincaré maps showing period-doubling bifurcations when the system is forced at $\omega=1, v=0.1$ at increasing amplitudes.

For this purpose, we consider the system (3) subjected to a small forcing in the high-Reynolds limit, $\bar{q}$ and $v$ being of the same order (say $\epsilon$ ). Equations (3) are now rearranged as

$$
\begin{aligned}
& \frac{d U}{d t}=U\left(1-U^{2}\right)-K u|u|+\epsilon\left(q^{\prime} \cos \omega t-v^{\prime} U\right), \\
& \frac{d u}{d t}=K U(|u|+\sigma)-\hat{v} u,
\end{aligned}
$$

where $\bar{q}=\epsilon q^{\prime}, v=\epsilon v^{\prime}$ and $\epsilon \ll 1$. We have already seen that, with the choice made for $K$ and $\hat{v}$, the unperturbed part in (6) and (7) possesses a homoclinic orbit. Following the procedure described in detail by Guckenheimer and Holmes, ${ }^{5}$ the separation between the stable and unstable manifolds of the perturbed system, measured normal to the unperturbed homoclinic orbit, is proportional to the Melnikov function $M$, which for the present problem takes the form

$$
\begin{aligned}
& M\left(\tau, v^{\prime}, q^{\prime}, \omega\right) \\
& =-\int_{-\infty}^{\infty} K U_{0}(t)\left[\left|u_{0}(t)\right|+\sigma\right]-\hat{v} u_{0}(t) \\
& \quad \times\left[q^{\prime} \cos \omega(t+\tau)-v^{\prime} U_{0}(t)\right] A(t) d t,
\end{aligned}
$$




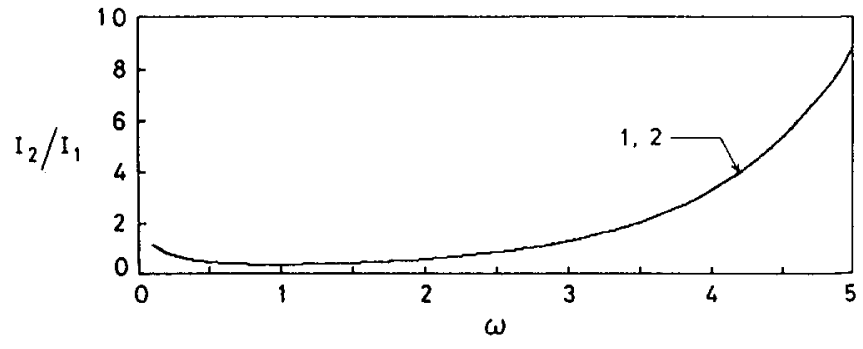

FIG. 8. The ratio of integrals in the Melnikov function, showing collapse for two different starts at $(0.259,0.016),(0.879,0.350)$, respectively, in the $(U, u)$ plane.

where

$$
\begin{aligned}
A(t)= & \exp \left(-\int_{0}^{t}\left[1-3 U_{0}(s)^{2}+K U_{0}(s) \operatorname{sgn} u_{0}(s)\right.\right. \\
& -\hat{v}] d s) .
\end{aligned}
$$

The subscript 0 on $U$ and $u$ denotes that these variables are evaluated along the unperturbed homoclinic orbit, and $\tau$ is a variable that denotes the phase of the Poincare section. Basically we stand at a point on the homoclinic orbit and watch how the separation between the manifolds changes in the Poincare sections at different phases.

The integral in (8) can be expressed as the sum

$$
M\left(\tau, v^{\prime}, q^{\prime}, \omega\right)=-q^{\prime} I_{1}(\omega) \cos (\omega \tau+\beta)+I_{2} v^{\prime},
$$

from which it follows that $M$ has simple zeros provided

$$
q^{\prime}>\left[I_{2} / I_{1}(\omega)\right] v^{\prime} \text { or } \bar{q}>\left[I_{2} / I_{1}(\omega)\right] v .
$$

This expression gives the minimum forcing amplitude required to get homoclinic tangles, and therefore chaos, for a given forcing frequency $\omega$ and $v(\ll 1)$.

In the present case, $I_{1}(\omega)$ and $I_{2}$ are numerically determined by selecting some initial point on the homoclinic orbit and integrating in forward and backward directions. The integration is found to converge rapidly. Although $I_{1}$ and $I_{2}$ individually depend on the initial point, the ratio $I_{2} / I_{1}$ does not, ${ }^{59}$ and is easily computed (Fig. 8). It is seen that the minimum forcing amplitude $\bar{q}_{\text {min }}$ required to induce chaos occurs at a forcing frequency $\omega \approx 1$.

This result demonstrates that chaos in the system (3) persists in the limit of high Reynolds number, the forcing required to induce chaos going simultaneously to zero in the limit-a behavior that we have seen is characteristic of boundary layer flows.

We now turn our attention to the important problem of identifying the range of $v$ and $\bar{q}$, where the system possesses chaotic solutions. This purpose is best served by computing the Lyapunov characteristic exponent.

\section{B. Lyapunov characteristic exponent (LCE)}

This number is a measure of the average divergence rate of two trajectories close to each other at the start, and is defined as
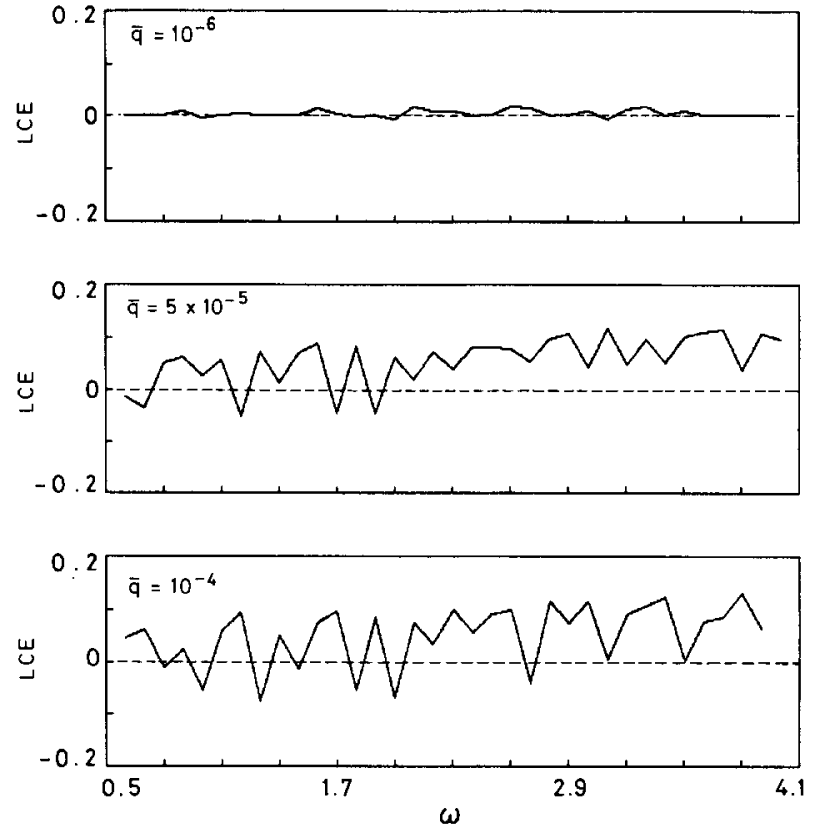

FIG. 9. Dependence of the Lyapunov exponent on $\omega$ and $\bar{q}$ at $v=0$.

$$
\lambda=\lim _{\substack{t \rightarrow \infty, d(0) \rightarrow 0}} \ln \left(\frac{d(t)}{d(0)}\right),
$$

where $d(t)$ denotes the separation between two orbits at time $t$. In general, a system with $n$ degrees of freedom has $n$ characteristic exponents, and if at least one of them is positive then the system may be taken to be chaotic. We have used the method of Wolf et al. ${ }^{68}$ to estimate the largest exponent; as the exponent associated with time is always zero, the largest is just the larger of the remaining two. These, in general, show a slow variation for small averaging times (typically $<500$ ). Conclusions drawn below are based on LCE's that have reached a more or less steady state. Since the LCE represents orbital stability, the accuracy of the numerical integration scheme employed for its determination is very important, and an absolute accuracy of at least $10^{-5}$ at output interval of 1 was essential for obtaining reliable estimates.

In general, the LCE depends on $v, \bar{q}$, and $\omega$; as an example, Fig. 9 shows it as a function of $\omega$ for three values of $\bar{q}$ at $v=0$. In this figure, $\omega$ is varied in steps of 0.1 over the range $0.6-4.0$ and successive points are joined by a straight line. It is seen that for a given $\bar{q}$, the LCE is positive in certain frequency bands, i.e., the model is more "receptive" at certain frequencies (using the word in the sense of Morkovin ${ }^{69}$ ), a well-known feature of many open flows. Further, it is seen that even for $\bar{q}=10^{-6}$, there are frequency bands where the LCE is positive, suggesting that the system is chaotic even at the smallest forcing, although there can be no chaos when the forcing vanishes; at higher levels of $\vec{q}$ there is a tendency to saturation. Similar variations of the LCE are observed at higher values of $v$ also, but the range of $\omega$ over which the LCE is positive narrows down, and a higher forcing amplitude is required to induce 


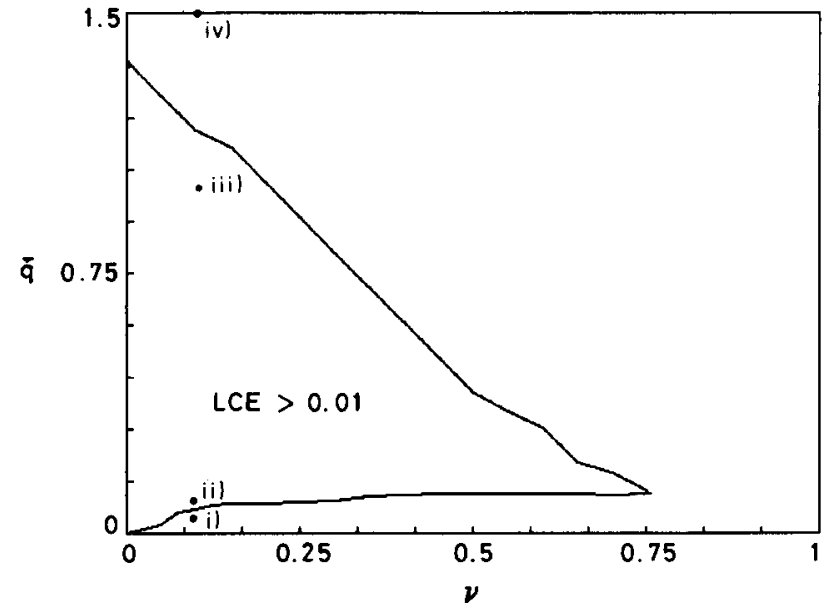

FIG. 10. Boundary separating chaotic and nonchaotic regimes in the system.

chaos. The latter point is brought out in Fig. 10 where the boundary separating LCE $>0.01$ from smaller and negative values, irrespective of forcing frequency, is shown. This diagram has been prepared from solutions in which $v$ is varied in steps of 0.025 for $v \leqslant 0.2$ and of 0.05 for larger $v$, and $\omega$ is varied in steps between 0.025 to 0.1 in the frequency range where the system is found to be most sensitive (e.g., 0.6 to 4.0 at $v=0$, and 0.7 to 1.25 at $v=0.25$ ). Because of the difficulty of accurately estimating the LCE, we have adopted the operational definition that the system is chaotic whenever LCE exceeds 0.01 . It is seen that the boundary in Fig. 10 turns back on itself, the region between the upper and lower curves covering the chaotic regime for the system. The lower curve depicts the minimum forcing required to induce chaos, and may be thought of as mimicking the known dependence of transition Reynolds number $R_{t}$ on the external disturbance level (seen in Fig. 2). Above the upper curve the system exhibits forced periodic oscillations, much as the wake behind a cylinder does if the cylinder is itself oscillating with large amplitudes, ${ }^{70}$ the system in this case may be regarded as exhibiting relaminarization (by "domination," as identified in Narasimha and Sreenivasan ${ }^{17}$ ). It is interesting to note that, as $v$ increases, the lower and upper curves approach each other and there is a value of $v(\approx 0.76)$ above which no chaos is possible according to the present criterion; this number may be thought of as corresponding to the lowest possible transitional Reynolds number $R_{t \text { min }}$, a concept for which there is considerable observational support. ${ }^{11}$

Some additional data on the frequency dependence of the sensitiveness of the system are shown in Fig. 11. Here the "most dangerous" frequencies, i.e., those at which the LCE first exceeds 0.01 as the forcing amplitude is increased, are plotted against $v$. This figure shows that for smaller $v$, the system responds to a wider frequency range centered around $\omega \approx 1$. This conclusion is consistent with results from the Melnikov analysis carried out above.

One issue that is yet to be resolved is whether the

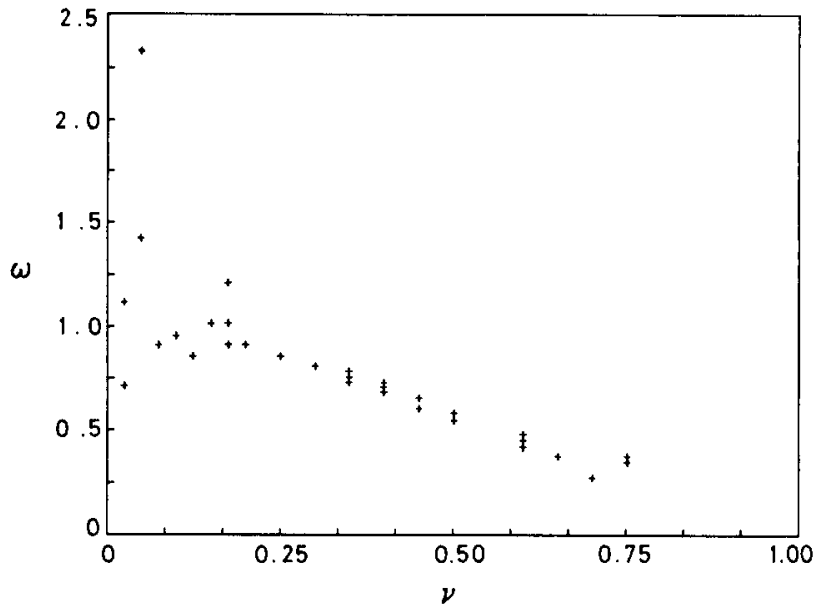

FIG. 11. The most dangerous or receptive frequencies (i.e., those yielding positive Lyapunov exponents at the lowest forcing amplitude).

boundary of the chaotic regime in Fig. 10 is a fractal, as Moon $^{71}$ has suggested in his study of a two-state mechanical oscillator.

\section{Time series, correlations, and spectra}

The conclusions based above on LCE are confirmed by examination of time series, correlations, and spectra. Figure 12 shows time series of $U$ at $v=0.1$ and at various forcing amplitudes motivated by the analysis of Sec. IV B. At $\bar{q}=0.05, U$ is periodic (as is $u$, not shown), whereas at $\bar{q}=0.1$ they appear to be nonrepeating, suggesting chaos. These forcing levels are, respectively, in the periodic and chaotic regions shown in Fig. 10. At $\bar{q}=1$, both $U$ and $u$ show chaotic behavior. At $\bar{q}=1.5$ periodicity is prominent, the system now clearly being in the forced oscillation regime of Fig. 10.

Some traces of the combination signal (2) for various values of the associated parameters are shown in Fig. 13. It

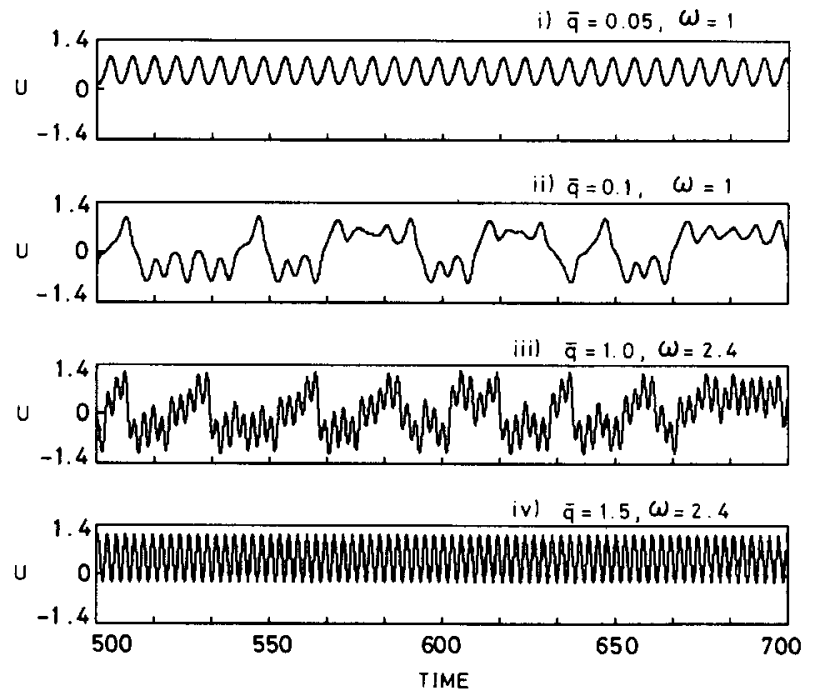

FIG. 12. Time series of $U$ at various periodic forcing levels, $v=0.1$. 


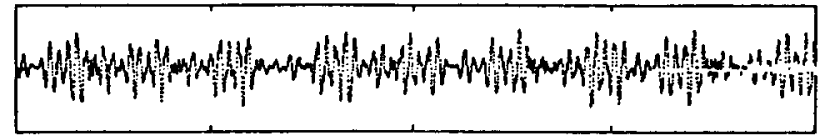

(a)

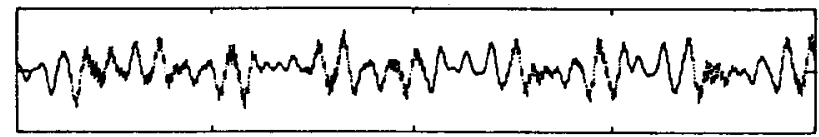

(b)

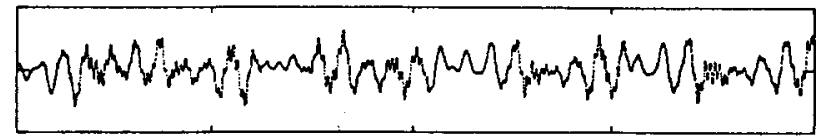

(c)

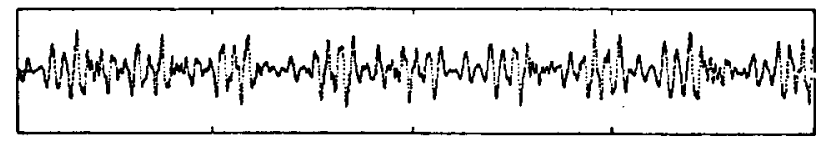

(d)

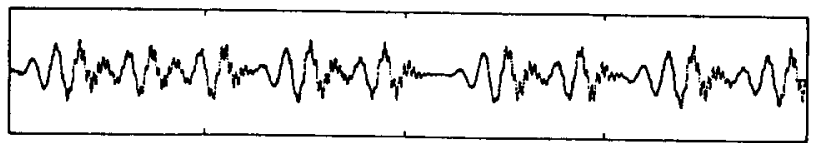

(e)

FIG. 13. Examples of velocity traces using the linear combination (2).

will be seen that these traces are not unlike velocity fluctuations characteristic of intermittent and fully turbulent flows.

The autocorrelations and spectra confirm the conclusions drawn from an examination of the LCE, and so will not be cited here (see Ref. 59).

\section{ROUTES TO CHAOS: PERIODIC FORCING}

In discussing routes to chaos, two different tracks in the $(\bar{q}, v)$ plane of Fig. 10 turn out to be of interest. In the first, the forcing amplitude $\bar{q}$ is gradually increased at a fixed value of $v$, as already described in Sec. III through an illustrative example. The second track, with fixed $\bar{q}$ but decreasing $v$, is more relevant to the fairly common fluiddynamical situation in which the level of external disturbance remains the same while some system parameter such as the Reynolds number increases continuously (for example, flow in a boundary layer developing downstream in a turbulent or acoustically excited environment, say in a wind tunnel or on an aircraft). Increasing Reynolds number in the flow corresponds roughly to decreasing $v$ in Eq. (3a); it is therefore interesting to understand the route to chaos with decreasing $v$. This track can be qualitatively different from the first because of a decrease in the natural frequency of oscillation of the unforced system as $v$ decreases (which is similar to the trend exhibited by Tollmien-Schlichting instability in the boundary layer). ${ }^{60}$ Thus if the forcing frequency is held constant while $v$ de- creases, the ratio of the natural to the forcing period varies continuously, and as a consequence the coupling between the forcing and the system as well; this could give rise to a more complicated transition scenario.

Before we describe the observed routes to chaos, a few words about the methodology adopted are necessary. In general, we have examined Poincare maps and computed the spectra and the Lyapunov exponents whenever necessary. When the forcing is periodic, it is usual to study the return map at the forcing period. An alternative procedure is to project the trajectory into the phase plane, cut it by a suitable line and examine the relationship between successive values of $U$ or $u$ as the trajectory crosses the line. This approach is adopted here sometimes, in particular, when the system undergoes period-doubling bifurcations; a judicious location for the section in the region where the orbits are well separated helps analysis. The equations are integrated with an absolute accuracy of $10^{-7}$ or better.

In drawing conclusions on the routes to chaos, it is important to be able to distinguish transient and steadystate behavior. As it was observed that the present system reaches a stationary state in most cases by $t=1000$, the equations have been integrated up to $t=2500$ and the results presented relate to the behavior in the last $500-1000$ units of time.

If an orbit returns to a position within an error tolerance of $10^{-4}$ regularly every $n$th time in a Poincare section, the behavior will be referred to as a $\mathbf{P} n$ cycle; thus $\mathbf{P} 1$ represents the classical limit cycle. If there are two (independent) frequencies in the solution (frequency-locked or quasiperiodic) motion takes place on a two-torus, easily identified by the arrangement of the points along a smooth closed curve in the Poincaré maps.

Last, as the emphasis here is on the qualitative changes in the structure of the solutions, Poincare maps and phase plots are often given without specifying the scales, which are adjusted in each plot to get the maximum clarity. In the spectrum, all components are normalized with respect to the tallest spike (when present), so that the relative strength of other spikes stands out.

\section{A. Increasing forcing amplitude}

We have already presented preliminary and illustrative results for Poincare maps along this track in Sec. III, where it was shown that with $v$ fixed at 0.1 and forcing frequency $\omega$ fixed at 1 , a cascade of period-doubling bifurcations occurs as $\bar{q}$ increases. For $\bar{q}<0.05$, quasiperiodic oscillations are seen for some starts; up to $\bar{q} \approx 0.07$ there is a P1 cycle. It turns out that four subsequent perioddoubling bifurcations could be clearly recognized; ${ }^{60}$ the first two convergence ratios

$$
\alpha_{n}=\left(\bar{q}_{n+1}-\bar{q}_{n}\right) /\left(\bar{q}_{n+2}-\bar{q}_{n+1}\right),
$$

for $n=1,2$, are, respectively, 5.66 and 4.5 , the latter being not far from the corresponding Feigenbaum number $(4.669 \ldots)$. The spectrum (Fig. 14) fills up at lower frequencies, illustrating the "slow" chaos referred to in Sec. I. 

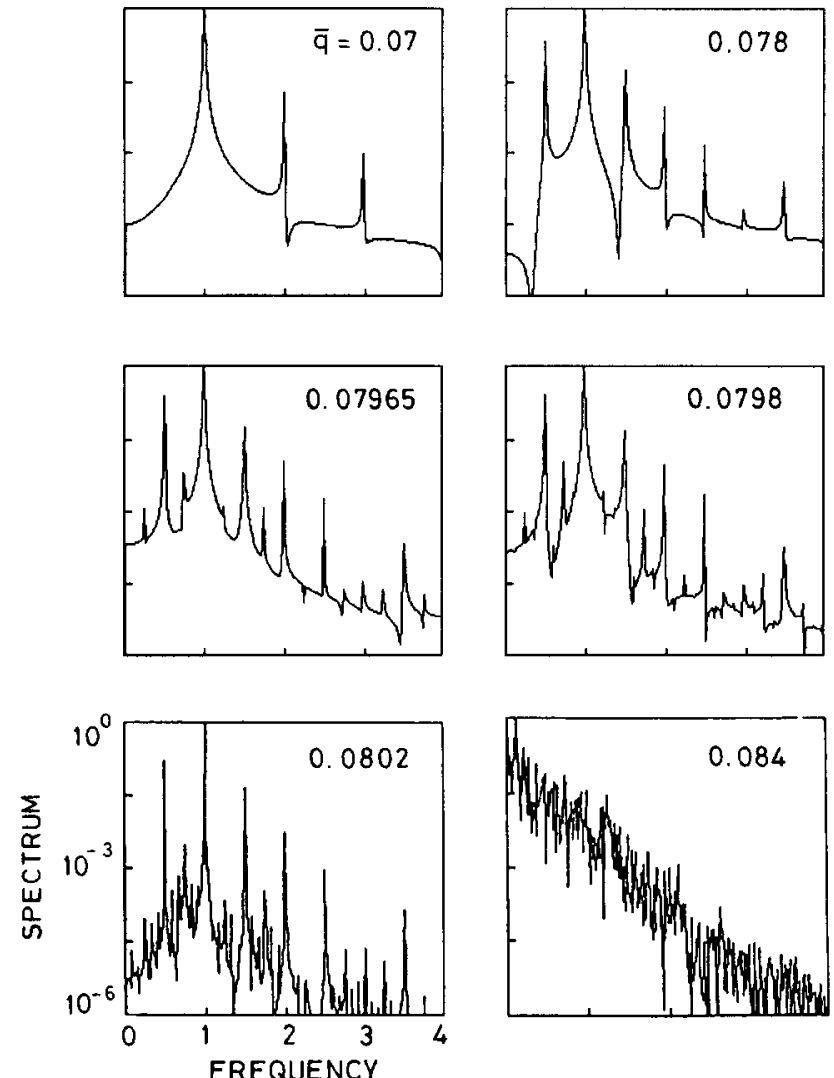

FIG. 14. Spectral evolution of $U$ along the "increasing $\bar{q}$ " track, at $v=0.1, \omega=1$. The first three period doublings can be easily identified; the low frequency spectrum fills rapidly for $\bar{q}>0.081$.

The above behavior is typical of this track, and could be indicating the Feigenbaum scenario, although convergence ratios of the kind quoted above cannot be taken as unambiguous evidence, in light of the work of Holmes. ${ }^{72}$

\section{B. Increasing Reynolds number}

Along this track the forcing frequency is an important parameter: we demonstrate this fact by considering two values of the frequency, namely, $\omega=0.7$ and 3.9 , which are, respectively, close to and far away from the natural frequency of the unforced system (approximately, 0.7 at $v=0.05$ and 1.05 at $v=0.11$ ).

\section{Forcing near the natural frequency: $\omega=0.7$, $\bar{q}=0.05$}

Figure 15 shows a selection of spectra as $v$ decreases. A detailed study ${ }^{60}$ reveals the following picture. For $0.128<v<1$ the steady state behavior is a P1 cycle at the forcing frequency $f_{1}(=\omega / 2 \pi)$. Between $v=0.127$ and 0.128 there is a Hopf bifurcation, resulting in a second frequency $f_{2}$ in the system for $v<0.128$. For $0.07<v<0.128, f_{1}$ and $f_{2}$ are not rationally related (at any rate not in small integer ratios), and the behavior is quasiperiodic. The frequency $f_{2}$ shifts as $v$ decreases, until at $v=0.068, f_{2}$ is rationally related to $f_{1}$, resulting in frequency locking. The frequency-locked state prevails until $v$ decreases to 0.043 (quasiperiodic solutions are also seen for some values of $v$ in this interval, e.g., at $v=0.06$ ).
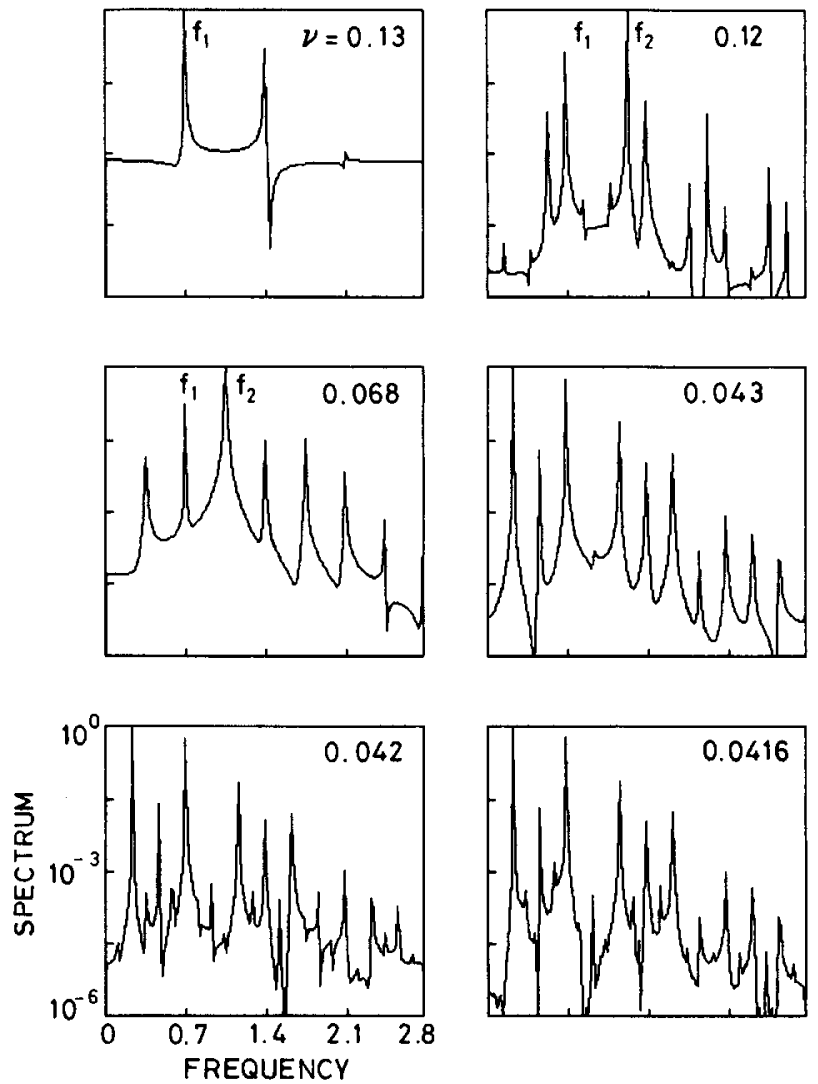

FIG. 15. Spectral evolution of $U$ along the "decreasing $v$ " track, at $\bar{q}=0.05, \omega=0.7$. Note the second spike at $f_{2}$ at $v \leqslant 0.12$; it first appears at $v=0.127$, changing in value from 0.200 at $v=0.127$ to 0.167 at $v=$ 0.068 , at which stage it locks with $f_{1}\left[=\left(\frac{2}{3}\right) f_{2}\right]$.

When $v$ decreases below 0.043 , the system undergoes period-doubling bifurcations with a convergence ratio of 4.2, suggesting a Feigenbaum scenario, ending up with chaos at $v=0.0414$.

When $v$ decreases further, the behavior is mainly chaotic, but there are narrow windows of periodicity buried in between, such as a P6 cycle at $v=0.0408$. At $v=0.0402$, the Poincare map resembles a cubic map, and takes more complicated shapes with a further decrease in $v$.

Thus the scenario here is quasiperiodicity $\rightarrow$ frequency locking $\rightarrow$ cascade of period-doubling bifurcations $\rightarrow$ chaos.

\section{Forcing at high frequency: $\omega=3.9, \bar{q}=0.05$}

In this case, the interaction between the forcing and $U$ is weak (or "receptivity" is low), and the system responds poorly, as can be seen from Fig. 16 where typical phase plots for $\omega=0.7$ and 3.9 are compared. There is a Hopf bifurcation around $v=0.128$, leading to quasiperiodic behavior for $0.0525<v<0.128$. Around $v=0.0525$, frequency locking takes place and prevails until $v$ decreases to 0.025 . For smaller $v$, the Poincaré maps develop a folded structure and the behavior is chaotic. This is confirmed by the positive Lyapunov characteristic exponents. ${ }^{60}$ There is no evidence to show the existence of period-doubling bifurcations so the route to chaos appears to be quasiperiodicity $\rightarrow$ frequency locking $\rightarrow$ sudden chaos. Such 


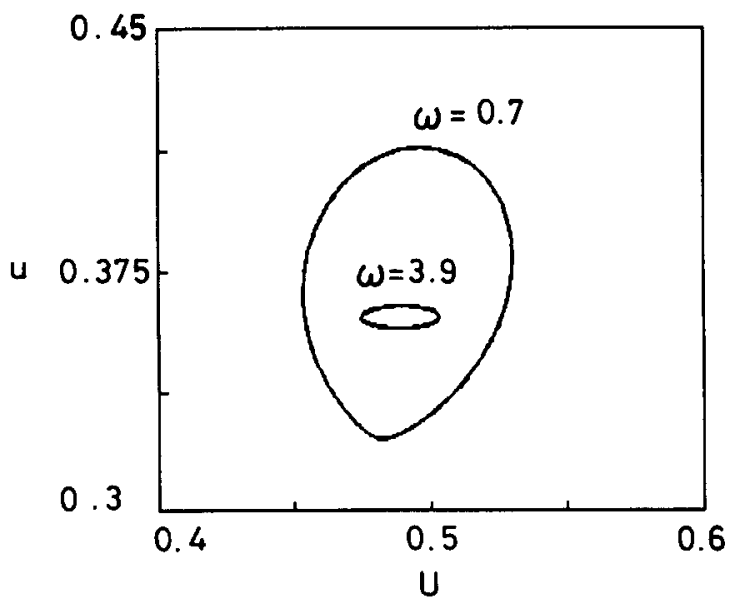

FIG. 16. Comparison of the response of the system to forcing of the same amplitude $\bar{q}=0.05$ at $\omega=0.7$ and 3.9 , respectively, near and away from the natural frequency.

a route has been observed by Gollub and Benson ${ }^{7}$ in convection experiments carried out in a cell. Spectra (Fig. 17) show that even though the behavior is chaotic, a strong periodic component is present in the solutions. In contrast to the previous case, there is no strong tendency for the low frequency region in the spectrum to fill up; furthermore, the milestones on the route to chaos occur at lower values of $v$.

It must be pointed out that there are difficulties sometimes in deciding whether the observed chaos is transient or steady. For example, for values of $v$ slightly above 0.025 solutions appear chaotic initially but later settle down to a periodic state. The outcome of some numerical experiments carried out to test the influence of accuracy of inte-
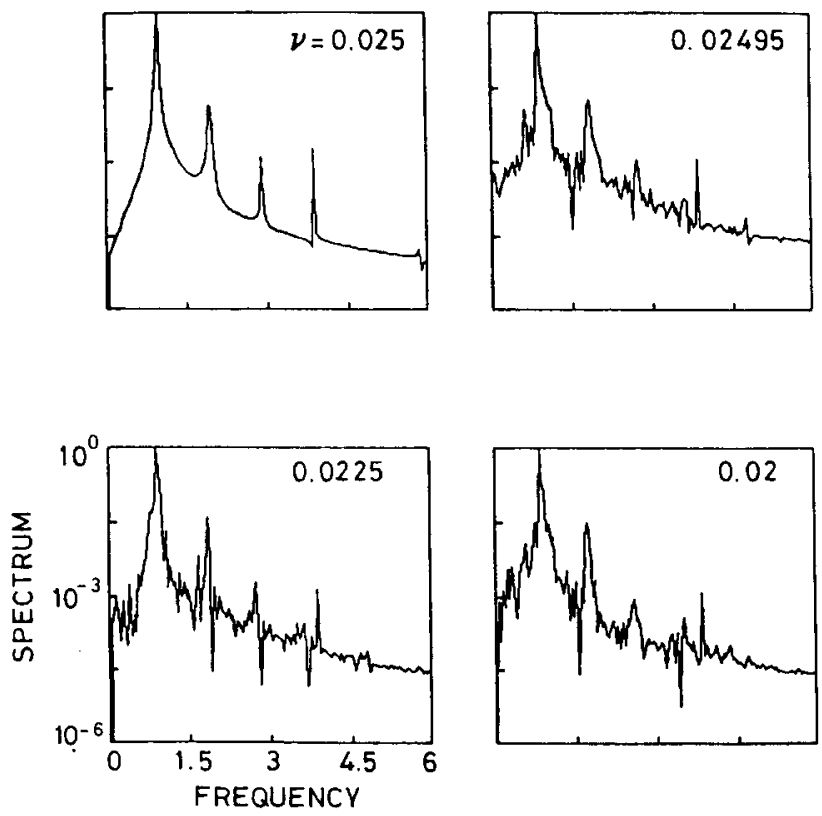

FIG. 17. Spectral evolution of $U$ close to transition along the "decreasing $v$ " track, with forcing at high frequency, $\bar{q}=0.05, \omega=3.9$, showing no strong tendency toward filling the low frequency side. gration and choice of initial condition on the transient period showed that for all levels of accuracy tested (up to $10^{-8}$ ), chaos persists for $v<0.025$, whereas for $v \geqslant 0.025$ results vary; no systematic dependence on accuracy of integration or starting point could be inferred except that for poorer accuracy (e.g., $10^{-6}$ ) chaos seems to persist even at $v=0.025$.

Comparison of these observations with the remarks made about the circle map (1) in Sec. I suggests that the value $v=0.025$ at $\bar{q}=0.05$ and $\omega=3.9$ is like $F=1$ in the circle map.

\section{COMBINED PERIODIC AND STOCHASTIC FORCING}

In practice, some noise is always present in any experiment, especially in open fluid flows, and this brings in some uncertainty in the inputs themselves. So there is considerable practical interest in understanding how the system behavior is modified when stochastic elements are present in the forcing.

In this section we study a few cases in which the forcing is largely periodic but includes a small stochastic term. This is intended to mimic the situation in a relatively quiet wind tunnel where a wave maker provides periodic excitation but the background contains some random disturbances, either naturally or otherwise (as, e.g., in Gaster's experiments, ${ }^{53,54}$ where noise is deliberately introduced). In the present work, the stochastic term is generated using algorithms described in Ref. 60, and is either a Gaussian pink noise (referred to as $\mathrm{N} 1$ ) with a peak in the spectrum near the natural frequency of the system (up to $\omega \cong 2$ ), or an amplitude-limited white noise (referred to as N2).

The method of Poincare sections, which proved to be a powerful tool in characterizing the nature of the solutions in Sec. V, is of little use in the present situation, as the driving noise contains a wide range of frequencies. Spectral analysis, on the other hand, has certain advantages, as it clearly brings out the relative importance of different modes and can give insight into the changes taking place in the solutions as the control parameter varies. So in this and the next section most conclusions are based on a study of the spectra. In order to get an idea of the actual development of different spectral components, the spectra below are given without normalization.

Results are presented in the following order. First a combination of $\mathrm{N} 1$ and a periodic component is considered, the choice of system parameter values being motivated by the results reported in Sec. V for deterministic forcing. Then transition under noise N2 is studied to assess the influence of the spectral distribution of the forcing on the scenario.

\section{A. Transition scenario with pink noise N1}

The result of increasing (the relative) noise level $\alpha \equiv q_{n} / \bar{q}$ on the spectral shape of $U$ at the parameter values $v=0.1, \omega=1$ and $\bar{q}=0.07$ is shown in Fig. 18. Data are sampled after an initial integration period of 1200 time units to remove transients. At these parameter values, the 

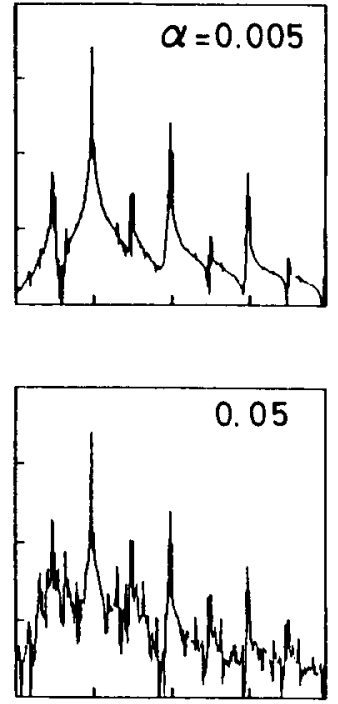

FIG. 18. Effect of increasing noise $\mathrm{N} 1$ in the forcing; $\bar{q}$ $=0.07, \omega=1$, and $v=0.1$. Only the first subharmonic bifurcation can be clearly recognized.

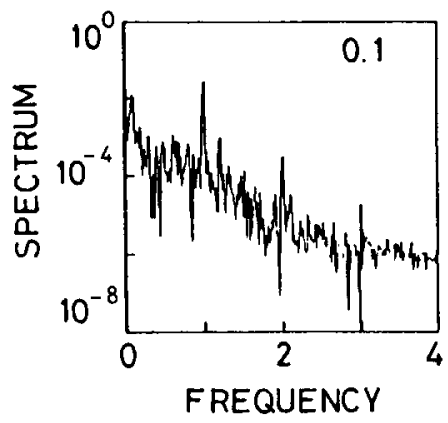

deterministic system has a P1 cycle and will experience the first period-doubling bifurcation if $\bar{q}$ increases (Fig. 7). Figure 18 shows that the introduction of an extremely small noise level (even $\alpha=0.001$ is enough) produces a P2 cycle, i.e., to cause the system to undergo a perioddoubling bifurcation. As $\alpha$ increases to 0.01 , the first subharmonic gains in strength; additional frequencies appear and grow as $\alpha$ increases. At $\alpha=0.1$, except for the peak at the periodic forcing, the spectrum is wide band suggesting chaos; so, while the $\mathrm{P} 1 \rightarrow \mathrm{P} 2$ bifurcation can be identified, higher period-doubling bifurcations are not clearly seen.

Figure 19(a) shows the effect of increasing the forcing at a fixed but low relative noise level $\alpha=0.01$. (For convenience of comparison with the results of Sec. IV, however, the diagrams are labeled in values of only the periodic forcing component $\bar{q}$.) In the range of $\bar{q}$ chosen, the deterministic system is in the regime of period-doubling bifurcations (there is a P1 cycle at $\bar{q}=0.07$ and a $P 16$ cycle at $\bar{q}=0.08$ if $\alpha$ is zero). At $\bar{q}=0.068$ and 0.07 , the spectrum suggests a noisy $\mathrm{P} 2$ cycle, and at $\bar{q}=0.072$ the behavior is quasiperiodic. At $\bar{q}=0.078$, a second perioddoubling bifurcation is suggested by the appearance of new frequencies near one-fourth the peak frequency. At $\bar{q}=0.08$, it is no longer possible to recognize any distinct subharmonics beyond the first and the spectrum at low frequency levels is almost wide band. Increasing the relative noise level advances the onset of chaos, as shown by Fig. 19(b) where all the parameters except $\alpha(=0.05)$ are the same as in Fig. 19(a). At all values of $\bar{q}$, the spectra
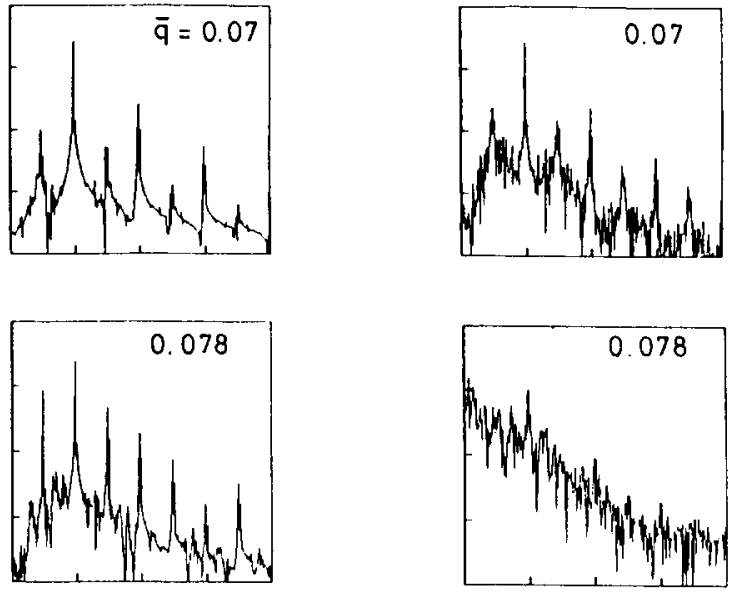

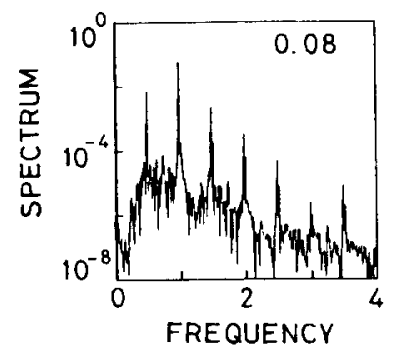

(a)

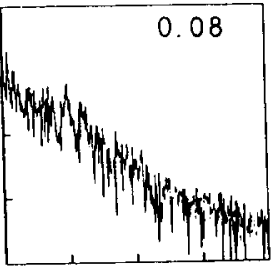

(b)
FIG. 19. Spectral evolution of $U$ with increasing forcing amplitude $\bar{q}$ at $\omega=1, v=0.1$ and (a) $1 \%$ and (b) $5 \%$ noise. Note the clear reflection symmetry around $\omega=1$ in the top spectrum of (b), very similar to the observation of Gaster. 53

cover a wider band; for $\bar{q}>0.078$, the spectrum is more or less continuous. These observations show that with increasing noise level, fewer periodic states are seen, a result that is in general agreement with the conclusion of Crutchfield and Huberman ${ }^{43}$ that noise deprives the system of certain (periodic) states. Interestingly, the spectra shown in Fig. 19(b) exhibit a symmetric development around the forcing frequency of precisely the kind that has been observed by Gaster ${ }^{53}$ in experiments on the boundary layer.

Next, the influence of noise when the deterministic system is in a frequency-locked state is considered. The parameter values chosen are $v=0.025, \bar{q}=0.05, \omega=3.9$, a set that has been shown in Sec. $V$ to be very close to the onset of chaos (behavior is chaotic for $v<0.025$ ). It will be seen from Fig. 20 that at $\alpha=0.001$ and 0.005 the spectra appear to be wide band. Interestingly, at $\alpha=0.01$ and 0.05 the spectra seem to have a stronger periodic component, suggesting that moderate noise levels have the tendency of stabilizing the system. However, if the noise level increases further, the stabilizing effect disappears and the spectra are wide band again. Noting that the system is on the verge of becoming chaotic, the noise does not exert as much influence here as when the system is in the period-doubling regime. 

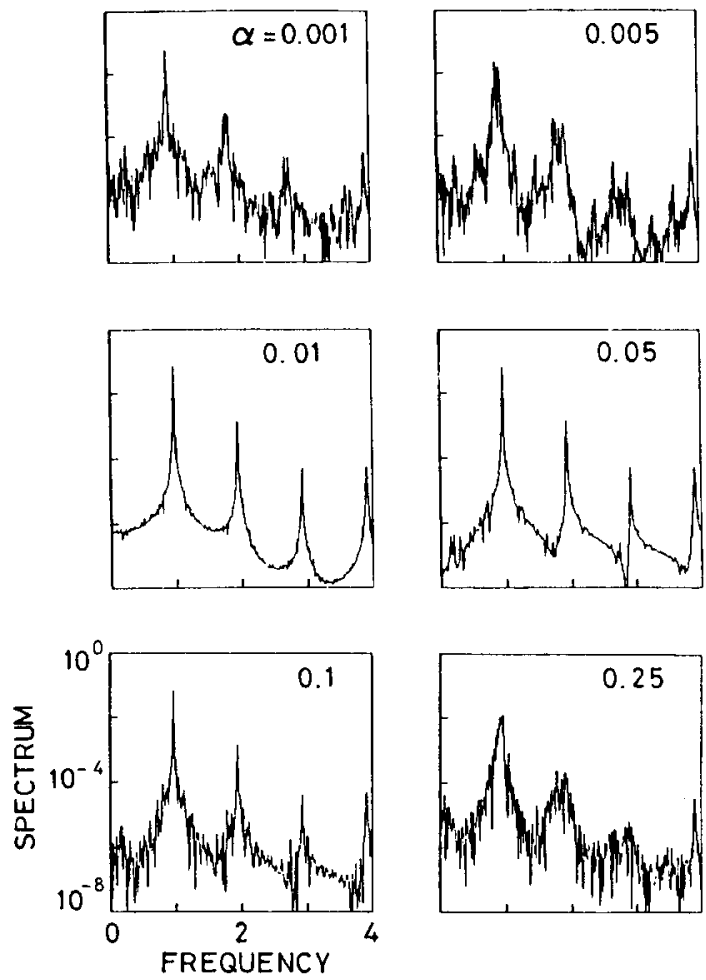

FIG. 20. Effect of addition of increasing noise levels $\mathrm{N} 1$ on spectral evolution when the deterministically forced system is frequency locked: $v=0.025, \bar{q}=0.05$, and $\omega=3.9$. The top two spectra are due to transient chaos, which vanishes in the presence of moderate levels of noise $(\alpha=0.01,0.05)$.

\section{B. Transition scenario under N2}

For comparison with N1, parameter values are the same as earlier except that N2 replaces N1. Figure 21 shows that at low values of $\alpha$, there are clear differences in the spectra, suggesting that the system is less receptive to noise $\mathrm{N} 2$ than to $\mathrm{N} 1$.

\section{TRANSITION UNDER PURELY STOCHASTIC FORCING}

This situation roughly corresponds to a flow development in a highly disturbed environment (e.g., turbomachinery). Studies on transition in different wind tunnels suggest that apart from the intensity of free-stream turbulence, its spectral distribution also plays an important role in transition. ${ }^{73}$ We examine this issue here briefly using forcing with $\mathrm{N} 1$ and $\mathrm{N} 2$.

\section{A. Transition under $\mathbf{N} 1$}

We first consider the case of constant forcing amplitude and varying $v$, which is more relevant for spatially developing flows. Selected spectra of $U$ for $q_{n}=0.02$ at different $v$ are shown in Fig. 22. There is no major change in the spectral shape for $v$ in the range 0.2 to 0.1 , with the peak at the angular frequency 1.208 and nearby prominent frequencies separated from the peak by integral multiples of 0.134 . However, there are some frequencies (e.g., 1.501 and 1.721) that do not follow this pattern; they have rel-
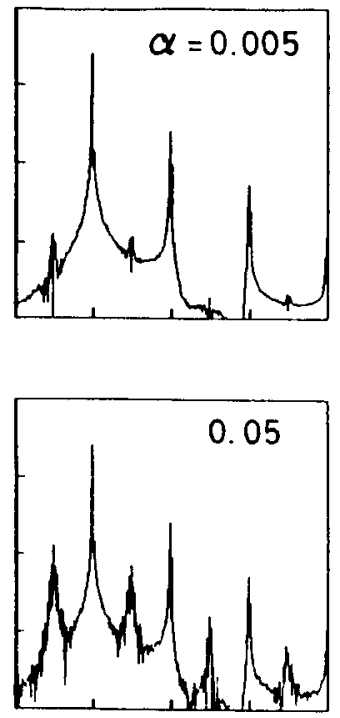

FIG. 21. Effect of noise $\mathrm{N} 2$ on spectral evolution under the same conditions as in Fig. 18.
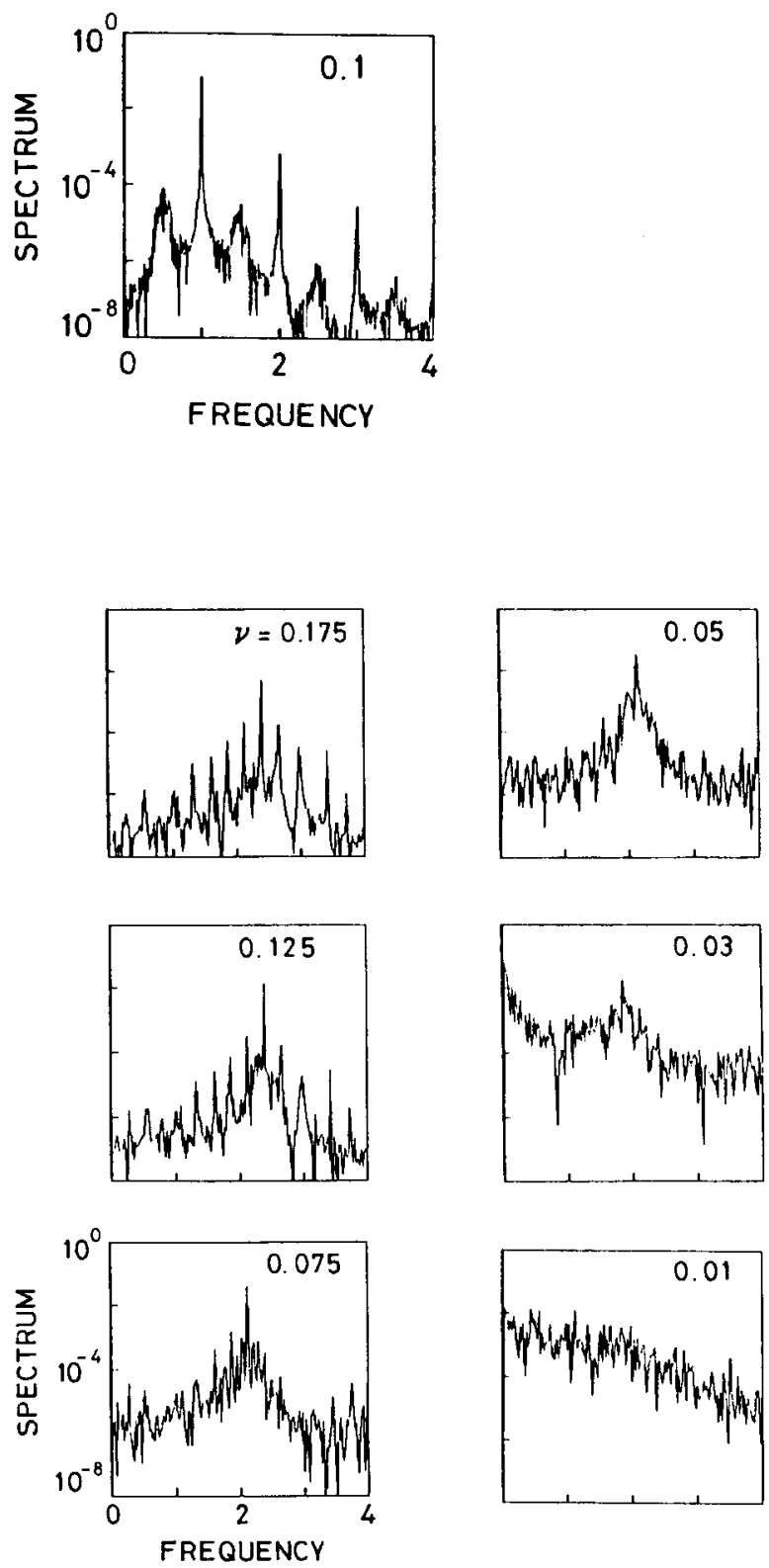

FIG. 22. Spectral evolution along the "decreasing $v$ " track with pure stochastic forcing by $\mathrm{N} 1$, with $q_{n}=0.02$. Note mainly noisy periodicity at high $v$, and the flat spectrum at low $v$. 

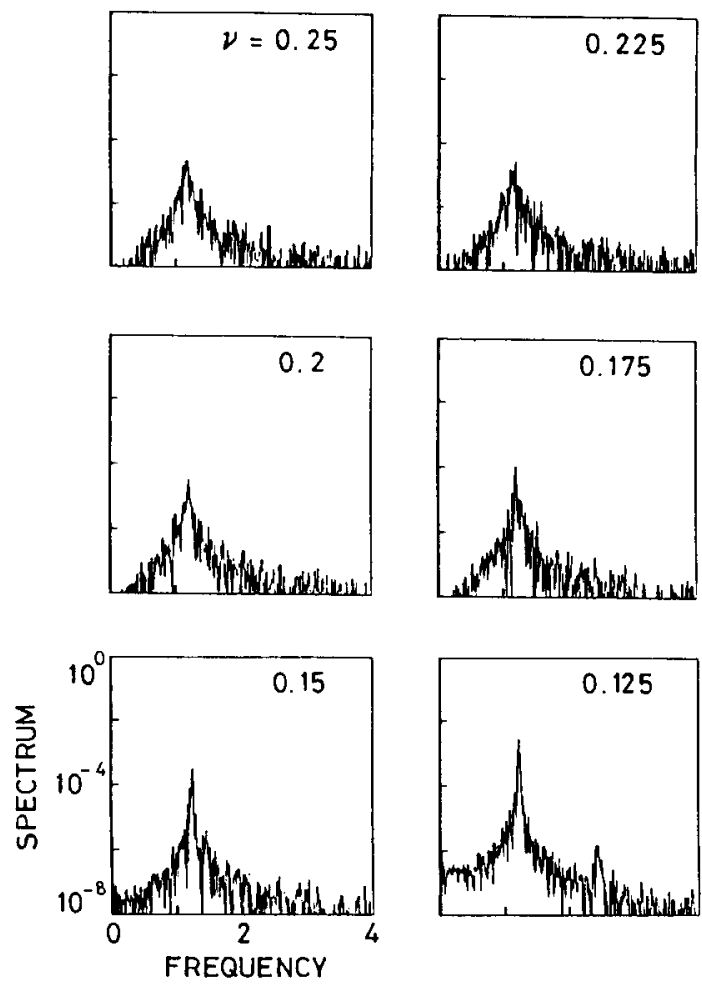

FIG. 23. As in Fig. 22, but with noise N2, $q_{n}=0.025$.

atively smaller energy. The overall conclusion is that the system is mainly frequency locked, although it may also be considered quasiperiodic since there is more than one independent frequency in the solution. As $v$ decreases to 0.075 , some major changes are seen, with the peak shifting to 1.074 and two new frequencies appearing between the earlier frequencies. These are almost equally spaced and presumably represent a higher state of frequency locking, i.e., the frequencies are related in the ratio of larger integers. With a further decrease in $v$ spectral lines broaden until slowly a wide band appearance is attained.

If $q_{n}$ increases for a fixed $v$ (not shown), the scenario is similar except that there is no indication of a higher level of frequency locking. ${ }^{60}$

\section{B. Transition under N2}

Again considering fixed $q_{n}$ and decreasing $v$, we see from Fig. 23 that for all values of $v$ shown the spectra are wide band; the major change as $v$ decreases is that the periodic component becomes stronger. There is no indication of frequency locking and it is difficult to discern any pattern in the spectra. Examination of time series, of which some representative examples are given in Fig. 24, provides some useful information. It is seen here that there are periods of low activity or small amplitude oscillations in between relatively active periods, giving an impression of intermittency; there is no indication of frequency locking. The intermittent behavior is more prominent when the unforced system cannot sustain a continuous oscillation, i.e., before the Hopf bifurcation.

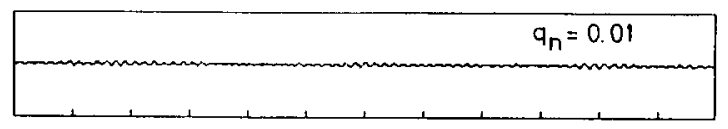

0.02
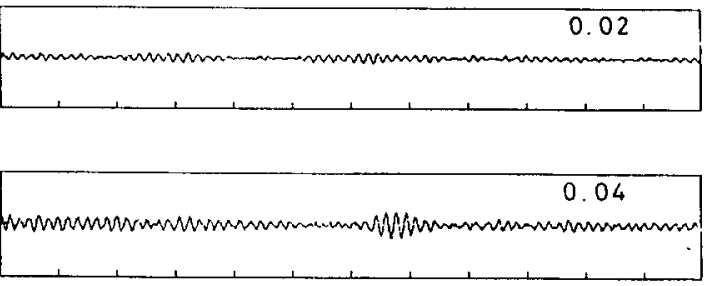

0.05

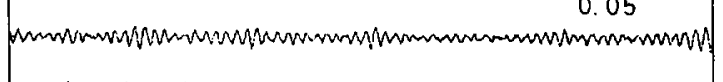

0 .

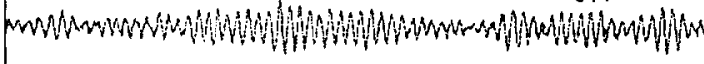

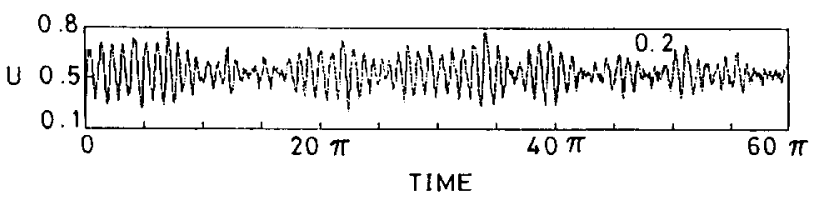

FIG. 24. The time series of $U$ at different forcing levels with noise $\mathrm{N} 2$, at $\nu=0.2$, showing intermittent-type behavior.

In none of these investigations of purely stochastic forcing was there any evidence of period-doubling bifurcations. As already mentioned, the period-doubling cascade is prominent especially when a special condition on the forcing frequency is met, i.e., when it is in the range of the natural frequency of the system. If there is no dominant frequency in the forcing, especially around the natural frequency of the system, as is often the case in highly disturbed open flows, the more likely routes to chaos appear to be those of intermittency and quasiperiodicity.

\section{DISCUSSION}

We have in previous sections argued that it is important to distinguish between different tracks in the $(\bar{q}, v)$ plane for the model (3), and that for spatially developing flows like the boundary layer on a plate the track at fixed $\bar{q}$ and decreasing $v$ may be more appropriate, especially when the disturbance environment can be considered to consist of free-stream turbulence or acoustic excitation. We have found the routes to chaos followed by the system when the forcing is periodic, stochastic or mixed.

The presence of a stochastic term in the forcing influences the route to chaos considerably. When the deterministically forced system is in the regime of period-doubling bifurcations, the number of bifurcations observed with the addition of the stochastic component decreases as the noise level increases; in a frequency-locked state, on the other hand, the system is less sensitive to the presence of noise that in moderate amounts appears to make the system 


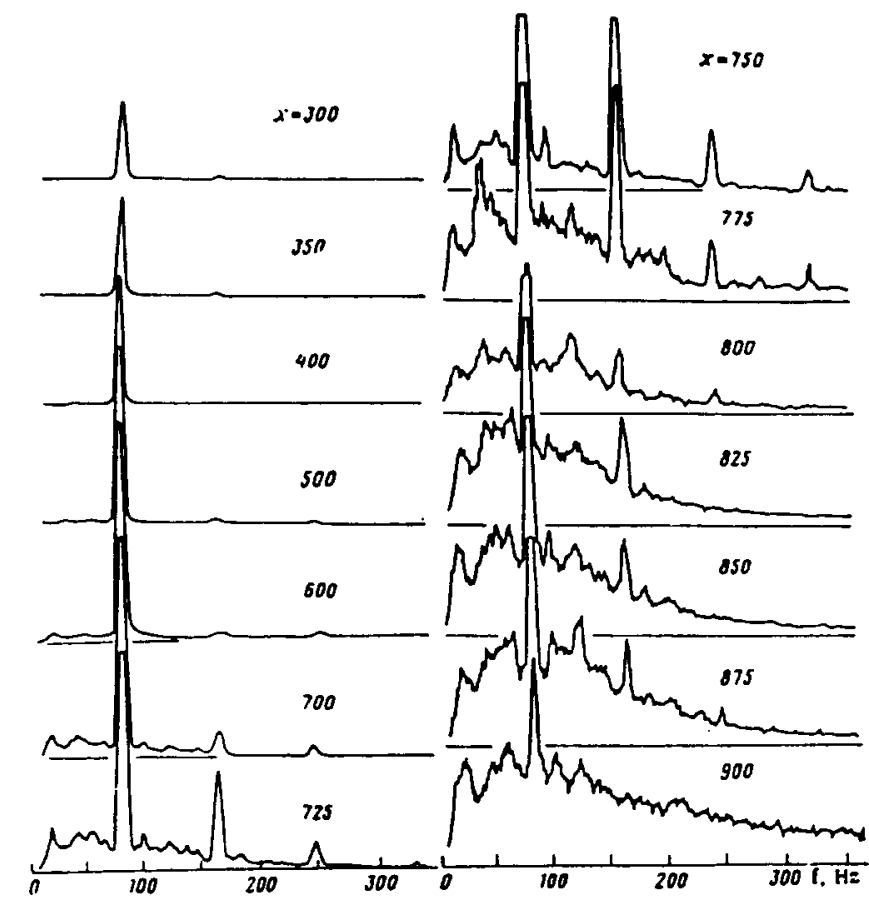

FIG. 25. Spectral evolution of velocity fluctuations in a boundary layer periodically excited at $81.4 \mathrm{~Hz}$ at a distance of $180 \mathrm{~mm}$ from the leading edge (from Ref. 74). Note the appearance of subharmonics at $700 \mathrm{~mm}$. Beyond $x=800 \mathrm{~mm}$ the low frequency spectrum changes little, but the high frequency end shows continuing development, even at $900 \mathrm{~mm}$.

more strongly periodic. Also, the spectral distribution of noise influences the transition process considerably.

Is there any correlation between what has been observed in this model and in real open flows? Making this comparison is rather difficult, chiefly because the forcing adopted in experimental studies (usually involving a wave maker at a fixed location) is not easy to reproduce in the model, and because the spectral distribution of other (presumably stochastic) disturbances always present in openflow facilities is rarely monitored. Nevertheless, it is interesting to examine, in particular, the work of Kachanov et $a l .{ }^{74,76}$ and Gaster, ${ }^{53,54}$ who have studied the spectral evolution of the fluctuations in a periodically excited flat-plate boundary layer (the latter with an additional stochastic component as well). Kachanov et al. ${ }^{76}$ report that the forcing amplitude is an important factor in determining the type of transition. In the most extensively reported case of relatively small amplitudes (typically less than $1 \%$ of the free-stream velocity), one subharmonic and several harmonics of the forcing frequency were observed before the appearance of a wide band spectrum; the low frequency end (in relation to the peak) of the spectrum developed more rapidly than that at high frequencies (Fig. 25). On the other hand, for large forcing amplitudes, the high frequency end underwent rapid changes and the low frequency end remained relatively unchanged.

These observations show some qualitative similarity with the model. For example, both Figs. 19 and 25 show a rapid rise in the energy content at low frequencies. In Fig. 19 we have already demonstrated that the system has a tendency to undergo period-doubling bifurcations (masked to some extent if there is a stochastic component in the forcing), and this leads to the rapid filling of the spectrum at the low frequency side. There is some evidence that Fig. 25 reveals a tendency for period-doubling bifurcations, although this is rendered fuzzy because of free-stream turbulence: we have seen that a small stochastic component in the forcing is enough to mask the later stages of the route. On the other hand the high frequency side appears to grow more rapidly than the low frequency end in a situation involving frequency locking in the model (Fig. 20), and it is plausible that the transition seen by Kachanov et al. at higher excitations is following this modified Ruelle-Takens scenario, again rendered fuzzy by free-stream turbulence.

In Gaster's experiments, a boundary layer in a quiet tunnel (free-stream turbulence $<0.01 \%$ ) was excited at a single point by a loudspeaker; sinusoidal, stochastic and mixed inputs were all tried. The observed spectral evolution suggests a clear distinction between "slow" and "fast" chaos. It was found that when the amplitude of the forced waves was large enough the flow was very sensitive to even weak broadband disturbances. Gaster remarks, in particular, on the symmetries in the observed spectra around the fundamental and the first subharmonic-of exactly the kind found in the model (see Fig. 19).

The model results show that the exciting frequency is an important parameter: if around the natural frequency the route to chaos is through period doubling, and if much higher the route is through frequency locking. It is not possible to say whether this is so in the real flows as well. In the experiments analyzed above the frequency was not varied over a wide range, but it would, however, not be surprising if the frequency played a less dominant role in a boundary layer, as the Tollmien-Schlichting instability is not limited to a single frequency but covers a continuous band.

\section{CONCLUSIONS}

We have presented a simple dynamical model for a rather peculiar driven nonlinear oscillator that possesses certain gross characteristics similar to those of many open fluid flows. The cascade process is built into the model through a single-step transfer of energy from large-eddy motion (providing one dependent variable) to small-eddy motion (a second variable). The model contains a parameter $v=R_{c} / R$ that is the analog of the inverse Reynolds number normalized by the value at the onset of linear instability. All unforced solutions of the model are nonchaotic. With periodic forcing the model is "receptive" to certain frequencies or frequency bands, and can be shown to exhibit chaos by demonstrating the existence of homoclinic tangles, horseshoes, and positive Lyapunov characteristic components. The value of $v$ at onset of chaos $v_{t}$ depends, in general, on both the amplitude $\bar{q}$ and frequency $\omega$ of the forcing. However, if the frequency is treated as a hidden variable, i.e., we look for the lowest forcing amplitude that induces chaos at some frequency, a boundary that encloses the chaotic regime can be found in the $(\bar{q}, v)$ plane. Along 
the lower boundary of this regime the forcing level required to trigger chaos goes to zero as $v \rightarrow 0$, though in its absence there is no chaos. Therefore the system has the property that chaos persists in the high Reynolds limit. (This property is consistent with a view of the laminar flow solutions in the asymptotic limit of Reynolds numbers tending to infinity as analogous to homoclinic orbits.) On the other hand, there in no chaos for $v>0.76$ for any frequency of the sinusoidal forcing considered; this value of $v$ corresponds to the minimum transition Reynolds number, below which turbulent flow is not possible. At extremely high values of the forcing [above the upper boundary of the chaotic regime in the $(\bar{q}, v)$ plane], the system is in forced oscillation, as may be expected if the flow relaminarizes by the mechanism of domination.

The routes to chaos exhibited by the system when subjected to periodic forcing are not unlike those observed in closed flow systems. With an additional stochastic component in the forcing, the transition processes are qualitatively similar to those observed in experimental studies of periodically and stochastically excited boundary layers, and are found to depend on both the intensity and spectrum of the forcing.

Further studies on variants of the model are continuing, to help understand subcritical transition, the onset of "fast" chaos following that of slow chaos and the role of spatial variations. While it is, of course, clear that not all flow chaos can be turbulence, it is hoped that the present model has shown how the physical mechanisms well known to operate in transitional and turbulent flows are not inconsistent with a view of turbulence as dynamical chaos.

\section{ACKNOWLEDGMENTS}

We are grateful to Professor A. Roshko for his constant encouragement and support, and have benefited from many discussions with him, Professor H. W. Liepmann, and Professor Phillip Holmes. The present version of this paper was prepared during a visit by RN to Cambridge, where he thanks Professor M. Gaster for interesting discussions and hospitality. The continued support of Professor A. Prabhu and Professor V. H. Arakeri at Bangalore is gratefully acknowledged.

This work has been supported in India by a grant from the Department of Science and Technology and in the U.S. by Contract No. N00014-85-K-0205 from the Office of Naval Research. The support received from the BOYS-CAST scheme of DST, and the Millikan Fund from Caltech, enabling visits to the United States by GSB and RN, respectively, were crucial for carrying out the project.

${ }^{1}$ D. Ruelle and F. Takens, Comments Math. Phys. 20, 167 (1971).

${ }^{2}$ S. E. Newhouse, D. Ruelle, and F. Takens, Comments Math. Phys. 64, 35 (1978).

${ }^{3}$ M. J. Feigenbaum, J. Stat. Phys. 19, 25 (1978).

${ }^{4}$ J. P. Eckmann, Rev. Mod. Phys. 53, 643 (1981)

${ }^{5} \mathrm{~J}$. Guckenheimer and P. Holmes, Nonlinear Oscillations, Dynamical Systems and Bifurcations of Vector Fields (Springer, Berlin, 1983).

${ }^{6} \mathrm{~S}$. Wiggins, Global Bifurcations and Chaos (Springer, New York, 1988).
${ }^{7}$ J. P. Gollub and S. V. Benson, J. Fluid. Mech. 100, 449 (1980).

${ }^{8}$ A. Libchaber, in Perspectives in Fluid Mechanics, edited by D. Coles (Springer, New York, 1988), pp. 103-112.

${ }^{9}$ H. L. Swinney and J. P. Gollub, Physica D 18, 448 (1985).

${ }^{10}$ A. S. Monin, Sov. Phys. Usp. 21, 429 (1978).

${ }^{11}$ M. Morkovin, ICASE Report No. 88-44, NASA Contractor Report No. $181693,1988$.

${ }^{12}$ B.-L. Hao, Chaos (World Scientific, Singapore, 1984).

${ }^{13}$ R. Narasimha, Curr. Sci. 56, 629 (1987).

${ }^{14}$ G. K. Batchelor, The Theory of Homogeneous Turbulence (Cambridge U.P., Cambridge, 1953).

${ }^{15}$ E. N. Lorenz, J. Atmos. Sci. 20, 131 (1963).

${ }^{16} \mathrm{~S}$. Corrsin and M. Uberoi, NACA Tech. Note 2124, 1950.

${ }^{17}$ R. Narasimha and K. R. Sreenivasan, Adv. Appl. Mech. 19, 221 (1979).

${ }^{18}$ C. Sparrow, The Lorenz Equations (Springer, Berlin, 1982).

${ }^{19}$ J. H. Curry, J. R. Herring, J. Loncaric, and S. A. Orszag, J. Fluid. Mech. 147, 1 (1984).

${ }^{20}$ V. Franceschini and C. Tebaldi, J. Stat. Phys. 25, 397 (1981).

${ }^{21}$ V. Franceschini and C. Tebaldi, Meccanica 20, 207 (1985).

${ }^{22}$ G. B. Schubauer and H. K. Skramstad, NACA Report No. 909, 1948.

${ }^{23}$ R. Govindarajan and R. Narasimha, NAL Report No. TM DU8901, 1989.

${ }^{24}$ G. L. Brown and A. Roshko, J. Fluid Mech. 64, 775 (1974).

${ }^{25}$ H. Aref, J. Fluid Mech. 143, 1 (1984).

${ }^{26}$ J. M. Burgers, Adv. Appl. Mech. 1, 171 (1948).

${ }^{27}$ R. J. Deissler, J. Stat. Phys. 40, 371 (1985).

${ }^{28}$ G. I. Sivashinsky, Annu. Rev. Fluid Mech. 15, 179 (1983).

${ }^{29}$ N. Aubry, P. Holmes, J. L. Lumley, and E. Stone, J. Fluid Mech. 192, 115 (1988).

${ }^{30}$ S. J. Kline, W. C. Reynolds, F. A. Schraub, and P. Runstadler, J. Fluid Mech. 30, 741 (1967).

${ }^{31}$ K. R. Sreenivasan, in Frontiers of Fluid Mechanics, edited by S. H Davis and J. L. Lumley (Springer, New York, 1985), pp. 41-67.

${ }^{32}$ C. W. Van Atta and M. Gharib, J. Fluid Mech. 174, 113 (1987).

${ }^{33}$ D. J. Olinger and K. R. Sreenivasan, Phys. Rev. Lett. 60, 797 (1988).

${ }^{34}$ R. Narasimha and G. S. Bhat, Curr. Sci. 57, 697 (1988).

${ }^{35} \mathrm{R}$. Narasimha, in Whither Turbulence, edited by J. L. Lumley (Springer, New York, 1990), pp. 13-48.

${ }^{36}$ L. Landau, in Collected Works, edited by D. ter Haar (Pergamon, Oxford, 1965), pp. 387-391.

${ }^{37}$ J. P. Gollub and H. L. Swinney, Phys. Rev. Lett. 35, 927 (1975).

${ }^{38}$ J. M. T. Thompson and H. B. Stewart, Nonlinear Dynamics and Chaos (Wiley, New York, 1987).

${ }^{39}$ V. I. Arnold, Am. Math. Soc. Transl. Ser. 2 46, 213 (1965).

${ }^{40}$ S. Ostlund, D. Rand, J. Sethna, and E. Siggia, Physica D 8, 303 (1983).

${ }^{41}$ Y. Pomeau and P. Manneville, Comments Math. Phys. 74, 189 (1980).

${ }^{42}$ J. A. Yorke and K. T. Alligood, Comments Math. Phys. 101, 321 (1985).

${ }^{43}$ J. P. Crutchfield and B. A. Huberman, Phys. Lett. A 77, 407 (1980).

${ }^{44}$ R. Kapral, M. Schell, and S. Fraser, J. Phys. Chem. 86, 2205 (1982).

${ }^{45}$ K. Matsumoto and I. Tsuda, J. Stat. Phys. 31, 87 (1983).

${ }^{46}$ P. Berge, M. Dubois, P. Manneville, and Y. Pomeau, J. Phys. Lett. 41, L341 (1980).

${ }^{47}$ M. Giglio, S. Musazzi, and U. Perini, Phys. Rev. Lett. 47, 243 (1981).

${ }^{48}$ A. Libchaber, C. Laroche, and S. Fauve, J. Phys. Lett. 43, L211 (1982).

${ }^{49}$ A. Libchaber and J. Maurer, in Nonlinear Phenomena at Phase Transitions and Instabilities, edited by T. Riste (Plenum, New York, 1982), pp. 259-286.

${ }^{50} \mathrm{H}$. W. Liepmann, in The Mechanics of Turbulence (Gordon and Breach, New York, 1964).

${ }^{51}$ P. S. Klebanoff, D. K. Tidstrom, and L. M. Sargent, J. Fluid Mech. 12, 1 (1962)

${ }^{52}$ T. Herbert, Annu. Rev. Fluid Mech. 20, 487 (1988).

${ }^{53}$ M. Gaster, in Turbulence and Chaotic Phenomena in Fluids, edited by T. Tatsumi (Elsevier, New York, 1984), pp. 99-106.

${ }^{54}$ M. Gaster, Proc. R. Soc. London Ser. A 430, 3 (1990).

${ }^{55}$ P. Huerre and P. A. Monkewitz, Annu. Rev. Fluid Mech. 22, 473 (1990).

${ }^{56}$ J. T. Stuart, J. Fluid Mech. 9, 353 (1960).

${ }^{57}$ A. A. Townsend, The Structure of Turbulent Shear Flows (Cambridge U.P., Cambridge, 1976)

${ }^{58}$ P. Holmes, Philos. Trans. R. Soc. London Ser. A 292, 419 (1979). 
${ }^{59}$ G. S. Bhat, R. Narasimha, and S. Wiggins, NAL Report No. PD DU8901, 1989.

${ }^{60}$ G. S. Bhat and R. Narasimha, NAL Report No. PD DU8903, 1989.

${ }^{61}$ A. Roshko, J. Fluid Mech. 10, 345 (1961).

${ }^{62}$ B. J. Cantwell and D. Coles, J. Fluid. Mech. 136, 321 (1983).

${ }^{63}$ K. R. Sreenivasan, P. J. Strykowski, and D. J. Olinger (private communication).

${ }^{64}$ J. M. Cimbala, H. M. Nagib, and A. Roshko, J. Fluid Mech. 190, 265 (1988).

${ }^{65}$ D. G. Bogard and W. G. Tiederman, J. Fluid Mech. 162, 389 (1986).

${ }^{66}$ V. K. Melnikov, Trans. Moscow Math. Soc. 12, 1 (1963).

${ }^{67}$ S. Wiggins, Introduction to Applied Nonlinear Dynamical Systems and Chaos (Springer, New York, 1990).

${ }^{68}$ A. Wolf, J. B. Swift, H. L. Swinney, and J. A. Vastano, Physica D 16, 285 (1985).
${ }^{69}$ M. Morkovin, Air Force Flight Dynamics Laboratory Report No. AFFDL-TR-68-149, 1969.

${ }^{70}$ C. H. K. Williamson and A. Roshko, J. Fluids Struct. 2, 335 (1988).

${ }^{71}$ F. C. Moon, Phys. Rev. Lett. 53, 962 (1987).

${ }^{72}$ P. Holmes, Phys. Lett. A 104, 299 (1984).

${ }^{73}$ H. U. Meier, U. Michel, and H.-P. Kreplin, in Perspectives in Turbulence Studies, edited by $\mathrm{H}$. U. Meier and P. Bradshaw (Springer, New York, 1987), pp. 47-70.

${ }^{74}$ Yu. S. Kachanov, V. V. Kozlov, and V. Ya. Levchenko, Fluid Dyn. 12, 383 (1977)

${ }^{75}$ Yu. S. Kachanov, V. V. Kozlov, V. Ya. Levchenko, and M. P. Ramanazov, in Laminar-Turbulent Transition, edited by V. V. Kozlov (Springer, New York, 1984), pp. 61-73.

${ }^{76}$ Yu. S. Kachanov and V. Y. Levchenko, J. Fluid Mech. 138, 209 (1984). 